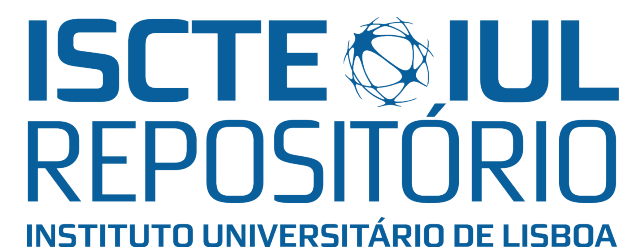

Repositório ISCTE-IUL

Deposited in Repositório ISCTE-IUL:

2019-03-26

Deposited version:

Post-print

Peer-review status of attached file:

Peer-reviewed

Citation for published item:

Gomes, O. (2009). Adaptive learning and complex dynamics. Chaos, Solitons and Fractals. 42 (2), 1206-1213

Further information on publisher's website:

$10.1016 /$ j.chaos.2009.03.077

Publisher's copyright statement:

This is the peer reviewed version of the following article: Gomes, O. (2009). Adaptive learning and complex dynamics. Chaos, Solitons and Fractals. 42 (2), 1206-1213, which has been published in final form at https://dx.doi.org/10.1016/j.chaos.2009.03.077. This article may be used for noncommercial purposes in accordance with the Publisher's Terms and Conditions for self-archiving.

Use policy

Creative Commons CC BY 4.0

The full-text may be used and/or reproduced, and given to third parties in any format or medium, without prior permission or charge, for personal research or study, educational, or not-for-profit purposes provided that:

- a full bibliographic reference is made to the original source

- a link is made to the metadata record in the Repository

- the full-text is not changed in any way

The full-text must not be sold in any format or medium without the formal permission of the copyright holders. 


\title{
Adaptive Learning and Complex Dynamics
}

\author{
Orlando Gomes ${ }^{1}$ \\ Escola Superior de Comunicação Social [Instituto Politécnico de Lisboa] \\ and Unidade de Investigação em Desenvolvimento Empresarial \\ - Economics Research Center [UNIDE/ISCTE - ERC].
}

- September 2008 -

${ }^{1}$ Address: Escola Superior de Comunicação Social, Campus de Benfica do IPL, 1549-014 Lisbon, Portugal. Phone number: + 3519334209 15; fax: + 351217 162 540. E-mail: ogomes@escs.ipl.pt. 


\begin{abstract}
In this paper, we explore the dynamic properties of a group of simple deterministic difference equation systems in which the conventional perfect foresight assumption gives place to a mechanism of adaptive learning. These systems have a common feature: under perfect foresight (or rational expectations) they all possess a unique fixed point steady state. This long term outcome is obtained also under learning if the quality underlying the learning process is high. Otherwise, when the degree of inneficiency of the learning process is relatively strong, nonlinear dynamics (periodic and aperiodic cycles) arise. The specific properties of each one of the proposed systems is explored both in terms of local and global dynamics. Two macroeconomic models are used to illustrate how the formation of expectations through learning may eventually lead to awkward long term outcomes.
\end{abstract}

Keywords: Adaptive learning, Nonlinear dynamics, Stability properties, Economic models.

JEL classification: C61, C62, D84, E32. 


\section{Introduction}

In the last few years, a large amount of literature has been produced on the subject of the formation of expectations under learning. This has emerged as a new paradigm that gradually replaced, in many areas of economic thought (and more specifically in the explanation of macroeconomic phenomena), the rational expectations hypothesis as formulated by Muth (1961) and applied to macroeconomics by Lucas (1972). Following the criticism of Marcet and Sargent (1989) on the too demanding nature of rational expectations (in what concerns the capacity of private agents in collecting information and using it optimally) it is today commonly accepted that a learning mechanism is able to better describe the true process of expectations formation by the agents in the economy.

Approaches to macroeconomic issues that assume expectations formed through learning comprehend a huge number of studies that one cannot cite here in a systematic and complete way. We refer the reader to some of the main references, as Evans and Honkapohja (2001, 2008), Honkapohja and Mitra (2003, 2006), Giannitsarou (2003) and Bullard and Mitra (2002). These studies focus on macroeconomic expectations in stochastic models, and they analyze stability and determinacy properties, inquiring essentially about the conditions that allow for convergence to a long run rational expectations outcome.

One of the most popular learning rules that is considered by the previous authors and others dealing with the same issues is the adaptive learning rule, under which agents act as econometricians, estimating, in each time moment, the value of the economic variables for the following periods of time. As new information arrives, expectations are updated and this process continues in time until eventually a long term state is reached; in this state, learning is no longer necessary (agents have already learned what they needed to form long run rational expectations). The argument is that an efficient learning process is a process in which learning leads to an asymptotic result of perfect foresight.

Instead of assuming perfectly efficient learning and therefore asymptotic rational expectations, one may imagine a process of learning where, as new information arrives allowing to improve expectations, there is also a memory loss or an imperfect knowledge component that is perpetuated, meaning that the long run state is one in which learning will continue indefinitely. This constant gain learning assumption (in opposition to the decreasing learning hypothesis that underlies the asymptotic rational expectations specification of learning), allows to reproduce long run nonlinear dynamics, which have been studied in the literature mainly in the context of overlapping generation models.

Some of the studies on macroeconomic expectations under adaptive learning that focus on the characterization of long run non conventional dynamic 
properties of the underlying systems include Bullard (1994), Cellarier (2006), Gauthier (2002), Hommes and Sorger (1998), Schonhofer (1999) and Sorger (1998). ${ }^{1}$ The main lesson that one withdraws from these studies is that as long as one assumes an asymptotically positive constant gain sequence, nonlinear dynamics will eventually arise and endogenous business cycles become an essential feature of the long term trajectories of the variables involved in the analysis.

In this text, we will explore the dynamic features of simple deterministic difference equation systems in which the conventional perfect foresight assumption gives place to a mechanism of adaptive learning. General nonlinear results are explored and macroeconomic examples will illustrate these results. The intention is to provide a framework for an easy identification of stability conditions in low dimensional systems with expectations formed under learning and in the presence of inefficiency elements that prevent the system to converge to a perfect foresight long run outcome. Besides finding stability conditions, the general settings and the macroeconomic models intend to characterize the behavior of the system out of the stable area. A global analysis is conducted, and one verifies that while some specifications imply period 2 cycles after a bifurcation is crossed, in other setups high-periodicity and a-periodic fluctuations are evidenced.

The richness of dynamic results emerges as the outcome of a bounded rationality setting that is perpetuated over time. In other words, the models to explore assume a less than perfect process of learning. Our argument is that an asymptotic constant learning outcome is more suitable to characterize real life conditions, in the sense that on one hand agents learn through time improving their knowledge about the phenomena in consideration, but, on the other hand, individuals also forget and lose part of the previously accumulated knowledge as time goes by. The following sentence, by Sobel (2002, p. 241), helps in clarifying our argument; about learning models, the author makes the following comment: 'Agents in these models begin with a limited understanding of the problems that they must solve. Experience improves their decisions. Death and a changing environment worsen them'.

In a learning context, the primary implicit assumption is that agents lack information. They are not able of automatically forming rational expectations because they have to apprehend the environment that surrounds them, and no agent is able to instantaneously solve a given problem without a time period devoted to learning. In this sense, the learning process is strictly related to the notion of time: it encompasses the time needed to make optimal decisions. The main issue is whether agents will be able to achieve a full understanding of the situation in order for their knowledge to

\footnotetext{
${ }^{1}$ The study by Cellarier (2006) departs significantly from the other ones because it studies the effects of constant gain learning over an intertemporal optimization growth model, rather than considering an OLG framework.
} 
be complete and therefore for them to be fully efficient in choosing among different options or predicting future values of given economic aggregates. In Sobel (2002), the fundamental requisites of an optimal learning process (i.e., a learning process leading to asymptotic rational expectations) are the following: $(i)$ a sensible starting point must exist (agents are only able to learn if the initial state is such that it allows to somehow follow a path that leads to a full understanding of the problem at hand); ( $i i)$ the environment should be stationary or it should change in a predictable way (otherwise, it is straightforward to understand that a non stationary environment will induce a never ending learning process); (iii) the costs to obtain and process information must not be too high (if they are, agents must weight the costs and benefits of gathering information and they will eventually stop to collect information before a complete understanding of the phenomena is accomplished).

One important feature of the systems that are analyzed throughout the paper is that the perfect foresight outcome (i.e., stability at the steady state level of the variable that is achieved under perfect foresight) does not require a complete convergence to long run rational expectations. The system may deviate from such long term outcome and still produce the desired stability result. This indicates that, if information is costly, agents should not make an effort to know everything about the problem at hand, but to locate in the point in which the result is the desired one and where there is the highest possible savings regarding the information collecting process. In other words, to act optimally does not necessarily pay: it allows to achieve an outcome that is obtainable with less than complete information, and therefore with lower costs. Finding the point of bifurcation (i.e., the point concerning to the amount of information / knowledge below which the stable outcome does no longer hold) introduces a non conventional notion of efficiency and rationality: who is the most rational agent, the one that gathers all the information to take the right decision or the one that knows that it is not necessary to acquire all the existing knowledge to arrive to the exactly same decision? This point is addressed in Orphanides and Williams (2007), who develop the notion of imperfect knowledge in the context of monetary policy problems. These authors realize that learning can be interpreted as a relatively modest deviation from rational expectations and therefore nest this as a limiting case.

The remainder of the paper is organized as follows. Section 2 explores five simple difference equation systems with adaptive learning in the formation of expectations. In all these models, long term nonlinear dynamics arise as the quality of learning decreases; in some systems, only period two cycles are identified, while in others cycles of higher periodicities and fully a-periodic cycles are generated. Section 3 explores two simple macroeconomic examples. The first regards the convergence of inflation to a specified target rate. Where under perfect foresight stability is guaranteed, under 
learning one may obtain a result of permanent fluctuation of the inflation rate around its target. The second example relates to the intertemporal optimization growth model; by inserting the state variable as an argument of the utility function and by assuming that expectations about the shadow price of capital are generated through learning, one encounters, also in this case, the possibility of nonlinear motion for both endogenous variables: the stock of capital and the control variable, which is the amount of consumption. Section 4 concludes.

\section{Five Models of Learning}

We develop five generic models in which expectations are formed through learning. In each one of them, we encounter nonlinear dynamic behavior that in some cases will correspond solely to period 2 cycles, while in more sophisticated difference equation systems chaotic motion is easily identified. The aim of this section is to provide some benchmark cases that are easily adaptable to the study of aggregate phenomena.

\subsection{Expectations over an exogenous value}

The first model to consider is a one dimensional system in which expectations about the next period level of an endogenous variable $x_{t} \in \mathbb{R}$ correspond to the contemporaneous value of an exogenous variable $y_{t} \in \mathbb{R}$, i.e., $E_{t} x_{t+1}=y_{t}$ ( $y_{t}$ given). To the exogenous variable we impose the condition of possessing a unique steady state $\bar{y}=\left\{\bar{y}: y_{t}=y_{t+1}\right\}$.

Expectations are formed through adaptive learning, in such a way that $E_{t} x_{t+1}=b_{t} x_{t}$, with $b_{t}$ an estimator based on past information. The estimator follows the dynamic rule $b_{t}=b_{t-1}+\sigma_{t} \cdot\left(\frac{x_{t-1}}{x_{t-2}}-b_{t-1}\right),\left(b_{0}\right.$ given $)$, as in Adam, Marcet and Nicolini (2006). Variable $\sigma_{t} \in[0,1]$ is central to the analysis and is generally known as the gain sequence. Decreasing gain is considered when one intends to translate the idea of efficient learning, i.e., when although agents learn through time, in the long run they are able to form expectations in a fully rational way and therefore they possess, asymptotically, perfect foresight. The decreasing gain sequence is such that variable $\sigma_{t}$ converges to zero as the system tends to the steady state. The dynamics underlying this type of learning gain can be expressed through the following rule: $\sigma_{t+1}=\sigma_{t} /\left(1+\sigma_{t}\right), \sigma_{0}$ given. The intuition underlying an efficient learning process is that in the long term predictions become accurate and therefore the agents do not need to learn anymore. Defining variable $\alpha_{t} \equiv 1 / \sigma_{t}$, one is able to better understand the concept of efficient learning; the variation of $\alpha_{t}$ in time can be interpreted as a learning increment; observing that $\alpha_{t+1}-\alpha_{t}=1$, one realizes that the increments are constant, i.e., in every time moment from $t$ to infinity, the representative agent is able 
to improve her decision making abilities.

An alternative interpretation of the gain sequence may be assumed, revealing a less than efficient learning result. Suppose now the following rule for the motion of the gain variable: $\sigma_{t+1}=\sigma_{t} /\left(1+\sigma_{t}+\delta \sigma_{t} \ln \sigma_{t}\right), \sigma_{0}$ given. In this case, we added to the above rule a memory loss term. Such a term is supposed to translate the idea that learning will occur as new information becomes available, but some of the previously stored information is also lost. Considering variable $\alpha_{t}$, we are now stating that $\alpha_{t+1}-\alpha_{t}=1-\delta \ln \alpha_{t}$, i.e., to the perpetual efficiency constant gain we subtract an information / knowledge depreciation or obsolescence term; as presented, this term indicates that diminishing marginal returns characterize the loss of previously stored knowledge.

The second specification implies the existence of two steady state points for the gain variable; $\bar{\sigma}=0$ is still a fixed point but it is no longer a stable fixed point, and therefore convergence to a perfect foresight outcome does not occur unless $\delta=0$. For $\delta>0$, the stable steady state is the point $\bar{\sigma}=\exp (-1 / \delta)$. Since we need to have $\sigma_{t} \leq 1$, then we impose the constraint $\delta \leq \exp (1)$.

The specification of the gain sequence under inefficiency, memory loss or imperfect knowledge allows to consider a long term constant gain setting, rather than decreasing gain. It is well known in the adaptive learning literature that constant gain allows for finding nonlinear dynamical behavior (as pointed out in the introduction). For some specific models we explore in what follows the properties of such nonlinear behavior.

Let us return to system $E_{t} x_{t+1}=y_{t}$. Replacing, in the estimator expression, the estimator by the ratio between the expected value of $x_{t}$ and the contemporaneous value of the variable, this yields the following system (presented one period ahead):

$$
\left\{\begin{array}{l}
x_{t+1}=\frac{y_{t}}{\left(1-\sigma_{t+1}\right) y_{t} / x_{t}+\sigma_{t+1} x_{t} / z_{t}} \\
z_{t+1}=x_{t}
\end{array}\right.
$$

Variable $z_{t}$ is defined as the value of variable $x_{t}$ in period $t-1$. System (1) reveals the existence of a unique steady state value for $x_{t}: \bar{x}=\bar{y} \cdot{ }^{2}$

Local dynamics are, in this simple case, straightforward to analyze. The most relevant characteristic is that long run local dynamics will depend only on the gain variable steady state value and not on $\bar{y}$.

Proposition 1 In the simple case in which expectations correspond to the contemporaneous value of an exogenous variable, the system is stable for $\bar{\sigma}<2 / 3$ and saddle-path stable otherwise. Point $\bar{\sigma}=2 / 3$ refers to a flip bifurcation point.

\footnotetext{
${ }^{2}$ We will find, as we explore other models, a relevant regularity: there is a coincidence between the rational expectations steady state and the steady state under learning.
} 
Proof. By linearizing system (1) in the vicinity of the steady state, one encounters

$$
\left[\begin{array}{c}
x_{t+1}-\bar{x} \\
z_{t+1}-\bar{x}
\end{array}\right]=\left[\begin{array}{cc}
1-2 \bar{\sigma} & \bar{\sigma} \\
1 & 0
\end{array}\right] \cdot\left[\begin{array}{c}
x_{t}-\bar{x} \\
z_{t}-\bar{x}
\end{array}\right]
$$

Conditions for stability are [let $J$ be the Jacobian matrix in (2)],

$1-\operatorname{Tr}(J)+\operatorname{Det}(J)=\bar{\sigma}>0$

$1+\operatorname{Tr}(J)+\operatorname{Det}(J)=2-3 \bar{\sigma}>0$

$1-\operatorname{Det}(J)=1+\bar{\sigma}>0$

The first and the third conditions are satisfied, independently of the value of $\bar{\sigma} \in(0,1)$. In the limit case, $\bar{\sigma}=0$, the system is on a bifurcation point, but one already knows that this corresponds to the rational expectations case. The second condition holds for $\bar{\sigma}<2 / 3$, and therefore this is the required condition for stability. When $\bar{\sigma}=2 / 3$, then $1+\operatorname{Tr}(J)+\operatorname{Det}(J)=0$ and the system will be over a flip bifurcation. For $\bar{\sigma}>2 / 3$, the second condition is violated, meaning that one of the eigenvalues of the Jacobian matrix falls outside the unit circle. In this case, one dimension is stable while the other one is unstable, and therefore saddle-path stability prevails

The result in proposition 1 may be depicted graphically. Noticing that $\operatorname{Tr}(J)=1-2 \bar{\sigma}$ and $\operatorname{Det}(J)=-\bar{\sigma}$, we establish the relation $\operatorname{Det}(J)=$ $[\operatorname{Tr}(J)-1] / 2$, which characterizes the dynamic behavior of the system. Figure 1 presents the location of the relation in a trace-determinant diagram; only a segment of such relation is displayed (in bold), corresponding to the points in which $\bar{\sigma} \in(0,1)$. Observe that the bifurcation line $1+\operatorname{Tr}(J)+\operatorname{Det}(J)=0$ is crossed at $\bar{\sigma}=2 / 3$.

$$
* * * \text { figure } 1^{* * *}
$$

From a global dynamics point of view, the construction of a bifurcation diagram indicates that, in this case, the bifurcation leads to a period 2 cycle for $\bar{\sigma}>2 / 3$. Figure 2 displays the bifurcation diagram for $\bar{y}=1$ (any other value conducts exactly to the same type of dynamics). The first 1,000 transient observations are excluded and the graphic is built for the subsequent 1,000 (this is the criterion used to draw the several diagrams presented in the paper). ${ }^{3}$ The main conclusion is that the long run value of $x_{t}$ departs from $\bar{y}$ as the gain variable assumes a high value. The lower quality of learning (translated on a higher $\bar{\sigma}$, that is related to stronger memory loss) implies that the long term value of the variable will be different from the rational expectations outcome, and this result is as much intense as the

\footnotetext{
${ }^{3}$ All the figures presented in this paper, with exception of figures $1,3,4$ and 5 , are drawn using IDMC software (interactive Dynamical Model Calculator). This is a free software program available at www.dss.uniud.it/nonlinear, and copyright of Marji Lines and Alfredo Medio.
} 
lower is the quality of learning (observing the figure, one directly perceives that after the bifurcation the average value of the variable falls, although to the right of $\bar{\sigma}=2 / 3$, the larger value assumed by the variable in the period 2 cycle is always above the steady state value).

$$
\text { ***figure } 2^{* * *}
$$

As referred in the introduction, a relevant result of the learning analysis consists in evaluating how much information the agent needs to attain a long term result identical to the one corresponding to the perfect foresight case. If information is costly and thus the agent withdraws utility from avoiding searching for information, the best outcome is the one in which the collected information is just enough for the agent to attain $\bar{\sigma}=2 / 3$; a higher gain value does not allow to obtain the perfect foresight stable outcome, while a lower gain value simply implies that the agent is wasting resources to obtain exactly the same outcome.

\subsection{A one-dimensional linear model}

Our second specification involves a unique variable $x_{t} \in \mathbb{R}$ and a parameter $a$ that may assume any value in the real line except 1 . The system is linear and takes the form:

$$
E_{t} x_{t+1}=a x_{t}-(1-a) \bar{x}, x_{0} \text { given }
$$

In equation (3), $\bar{x}$ corresponds to the steady state value of $x_{t}$. Under perfect foresight, it is straightforward to conclude that the system is stable for $a \in(-1,1)$ and unstable otherwise. The process of learning and particularly the eventual low quality of learning will change this result. Considering again the adaptive learning mechanism of the previous section, we arrive to the pair of equations

$$
\left\{\begin{aligned}
x_{t+1} & =\frac{(1-a) \bar{x}}{\left(1-\sigma_{t+1}\right)(1-a) \bar{x} / x_{t}+\sigma_{t+1}\left(x_{t} / z_{t}-a\right)} \\
z_{t+1} & =x_{t}
\end{aligned}\right.
$$

The steady state of the system is the same with and in the absence of learning, i.e., $\bar{x}$ (obviously, $\bar{z}=\bar{x}$ ).

Regarding stability, let us first look to the local dynamics results. The analysis requires separating two cases: (i) $a<1$; (ii) $a>1$. In both, the value of $\bar{x}$ is irrelevant for the stability outcome.

Proposition 2 In the one dimensional linear adaptive learning model, under condition $a<1$ the system is locally stable if $\bar{\sigma}<\frac{2(1-a)}{3-a}$. 
Proof. In the steady state vicinity, the following linear system is computed:

$$
\left[\begin{array}{c}
x_{t+1}-\bar{x} \\
z_{t+1}-\bar{x}
\end{array}\right]=\left[\begin{array}{cc}
1-\bar{\sigma}-\frac{\bar{\sigma}}{1-a} & \frac{\bar{\sigma}}{1-a} \\
1 & 0
\end{array}\right] \cdot\left[\begin{array}{c}
x_{t}-\bar{x} \\
z_{t}-\bar{x}
\end{array}\right]
$$

Applying stability conditions, one finds:

$1-\operatorname{Tr}(J)+\operatorname{Det}(J)=\bar{\sigma}>0$

$1+\operatorname{Tr}(J)+\operatorname{Det}(J)=2-\left(\frac{3-a}{1-a}\right) \bar{\sigma}>0$

$1-\operatorname{Det}(J)=1+\frac{\bar{\sigma}}{1-a}>0$

The first condition is satisfied for any admissible value of $\bar{\sigma}$. The same is true for the third condition under the imposed constraint on $a$. Condition number 2 holds as long as $\bar{\sigma}<\frac{2(1-a)}{3-a}$

Notice that a flip bifurcation occurs at $\bar{\sigma}=\frac{2(1-a)}{3-a}$ (i.e., one of the eigenvalues of $J$ equals -1 under the referred condition). Saddle-path stability holds for $\bar{\sigma}>\frac{2(1-a)}{3-a}$. Once again, we verify the relevance of the quality of learning. Efficient learning (i.e., $\bar{\sigma}$ low) conducts to stability; inefficient learning (i.e., $\bar{\sigma}$ high) can produce absence of stability (and, as we will see in the global dynamics analysis, it produces nonlinear motion).

Consider now the second case: $a>1$.

Proposition 3 In the one dimensional linear adaptive learning model, under condition $a>1$ the system is locally stable if $\bar{\sigma}<a-1$.

Proof. Consider again the stability conditions in the proof of proposition 2. For $a>1$, the second condition is always satisfied. The third condition requires $\bar{\sigma}<a-1$

The condition in proposition 3 implies as well that if $a>2$ then the system is stable independently of the long run value of the gain variable $\sigma_{t}$. Instability (two eigenvalues of the Jacobian matrix larger than 1 in modulus) arises for $\bar{\sigma}>a-1$. The point of bifurcation is associated to the condition $\bar{\sigma}=a-1$. In this case, a Neimark-Sacker bifurcation occurs, given that $\operatorname{Det}(J)=1$ and therefore a pair of complex conjugate eigenvalues with modulus equal to one will be obtained from solving the characteristic equation of $J$. Observe, one more time, that the lower is $\bar{\sigma}$, the more likely it is to obtain stability.

The results in propositions 2 and 3 are now analyzed graphically. Figure 3 presents the areas of local stability and instability in the space of parameters. The differences relatively to the perfect foresight case are evident. Now, we encounter stability where it did not exist in the perfect foresight case (i.e., for values of $a$ lower than -1 and above 2, independently of the value of $\bar{\sigma})$. For $a \in(-1,2) /\{1\}$, stability requires a relatively low value of $\bar{\sigma}$. 
Complementarily to figure 3, the graphical analysis may involve the relation between trace and determinant of the Jacobian matrix. The following relation is directly obtained: $\operatorname{Det}(J)=\operatorname{Tr}(J)-(1-\bar{\sigma})$. According to this expression, the relation between the trace and the determinant of $J$ is such that the dynamics of the model will be described by a line that is parallel to the bifurcation line $\operatorname{Det}(J)=\operatorname{Tr}(J)-1$, and that is located to the left of this last one. Let us take the two cases separately; figure 4 respects to the case $a>1$ [i.e., $\operatorname{Det}(J)>0$ ]; figure 5 assumes $a<1$ [i.e., $\operatorname{Det}(J)<0$ ].

$$
\text { *** figure } 4 \text { *** }
$$

Figure 4 draws the stability and instability results presented in proposition 3. For $\operatorname{Tr}(J)>2-\bar{\sigma}$ (and $a<1+\bar{\sigma}$ ), instability prevails. When $1-\bar{\sigma}<\operatorname{Tr}(J)<2-\bar{\sigma}$ (and $a>1+\bar{\sigma}$ ), the system falls in the stability region (the segment of the displayed line is inside the inverted triangle that represents, in the trace-determinant diagram, the area inside the unit circle).

In figure 5, the trace-determinant relation is presented for $a<1$. In this case, the bifurcation line $1+\operatorname{Tr}(J)+\operatorname{Det}(J)=0$ is crossed at $\operatorname{Tr}(J)=$ $-\bar{\sigma} / 2$ and $\operatorname{Det}(J)=-1+\bar{\sigma} / 2$. To the right of this point, stability holds. To the left of the presented point, the system is saddle-path stable. Note that as one goes up in the dynamics line (from the left to the right), the value of parameter $a$ decreases [observe, for instance, that $a=0$ implies $(\operatorname{Tr}(J), \operatorname{Det}(J))=(1-2 \bar{\sigma} ;-\bar{\sigma})$ and that $a=-1$ implies $(\operatorname{Tr}(J), \operatorname{Det}(J))=$ $(-1-(3 / 2) \bar{\sigma} ;-\bar{\sigma} / 2)]$.

$$
\text { *** figure } 5 \text { *** }
$$

Turning to the global dynamic analysis, one verifies that, differently from what occurred in the first proposed model, the area of absence of stability does not correspond solely to a region of cycles of periodicity 2. Other periodicities and full a-periodicity (possibly chaotic motion) are also found. Table 1 presents the largest Lyapunov characteristic exponent (LCE) of the system for various values of parameters $a$ and $\bar{\sigma}$ relatively to which chaotic motion is observed. ${ }^{4}$ LCEs are a measure of divergence of nearby orbits. In a two dimensional model as the one considered, the existence of (at least) one positive LCE is synonymous of divergence of orbits in a sense that one can talk about sensitive dependence on initial conditions (SDIC). In turn, the notion of SDIC is associated with the presence of chaotic motion (a same system produces completely distinct orbits for slightly different initial conditions). One observes that for various pairs $(a, \bar{\sigma})$ that in figure 3 correspond to regions of instability / saddle-path stability, chaos is found. Thus, irregular cycles are identified in a simple model with eventually low quality learning.

\footnotetext{
${ }^{4}$ LCEs are computed resorting also to the iDMC software.
} 
In figure 6 , the relation between parameters $a$ and $\bar{\sigma}$ is re-analyzed under a global dynamics perspective. The region of cycles is the one that was already identified as conducting to the absence of stability. Most of this area corresponds to cases of periodicity 2 , but cycles of higher periodicity and chaotic motion are observed for relatively high values of $\bar{\sigma}$.

$$
* * * \text { figure } 6 * * *
$$

We complete the global dynamic analysis presenting three additional figures: figures 7 and 8 display bifurcation diagrams for two different values of $a(a=0.8$ and $a=1.2)$ and taking $\bar{\sigma}$ as the bifurcation parameter. The path from stability to cyclical motion is evidenced. Figure 9 is the long term time series of variable $x_{t}$ (after excluding the 1,000 first iterations of the dynamic process) for a pair of values of $a$ and $\bar{\sigma}$ for which chaos is observed.

$$
\text { *** figures } 7,8,9^{* * *}
$$

\subsection{A one-dimensional nonlinear model}

In this sub-section, we develop one more one-dimensional expectations system (that, as the ones before, is transformed in a two-dimensional system once learning is introduced). Consider now

$$
E_{t} x_{t+1}=a x_{t}^{\phi}, x_{0} \text { given }
$$

Since relevant dynamics are obtained essentially for $a>0$ and $0<\phi<1$, we impose these two constraints. The steady state under perfect foresight is $\bar{x}=a^{1 /(1-\phi)}$. The same type of adaptive learning mechanism used before is again considered, in order to arrive to the following system:

$$
\left\{\begin{array}{l}
x_{t+1}=\left[\left(1-\sigma_{t+1}\right) x_{t}^{-(1-\phi)}+\sigma_{t+1} \cdot \frac{x_{t}}{a z_{t}}\right]^{-1 /(1-\phi)} \\
z_{t+1}=x_{t}
\end{array}\right.
$$

Once more, the coincidence between the steady state under perfect foresight and under learning is observed.

The interesting point about this model is that local dynamics are very similar to the dynamics found in the previous linear case. In the present formulation, the relevant parameters are $\phi$ and $\bar{\sigma}$; the value of $a$ is irrelevant for the dynamics.

Proposition 4 The one-dimensional nonlinear model with expectations formed under learning is locally stable if condition $\bar{\sigma}<2(1-\phi) /(3-\phi)$ holds. 
Proof. Computing the linearized system around the steady state one obtains:

$$
\left[\begin{array}{c}
x_{t+1}-\bar{x} \\
z_{t+1}-\bar{x}
\end{array}\right]=\left[\begin{array}{cc}
1-\bar{\sigma}-\frac{\bar{\sigma}}{1-\phi} & \frac{\bar{\sigma}}{1-\phi} \\
1 & 0
\end{array}\right] \cdot\left[\begin{array}{c}
x_{t}-\bar{x} \\
z_{t}-\bar{x}
\end{array}\right]
$$

Note that system (8) is identical to (5), except for the parameter that appears in the denominator of the fraction in the two elements of the first line of the matrix: it is now the parameter that introduces nonlinearity in the system. Because $\phi \in(0,1)$, we restrict the dynamic analysis to the case in proposition 2, i.e., the stability conditions are similar to the ones presented in the proof of such proposition and the only one that is violated in some point is $1+\operatorname{Tr}(J)+\operatorname{Det}(J)>0$, which now corresponds to $2-\left(\frac{3-\phi}{1-\phi}\right) \bar{\sigma}>0$; solving the inequality in order to $\bar{\sigma}$, the condition in the proposition is found

Given the similarities with the case of the previous section, we will not analyze this case in detail. Just keep in mind that a figure like figure 3 could be presented replacing parameter $a$ by $\phi$ and taking solely the region in which $\phi \in(0,1)$, and therefore a stable region would be found to the left of the bifurcation line and a saddle-path stability region would be encountered to the right of such line. Once again, stability vanishes as the long run value of $\sigma_{t}$ gets relatively high. A diagram similar to figure 5 could be presented with the segment of line that translates the dynamics of the system ranging from $(\operatorname{Tr}(J), \operatorname{Det}(J)) \rightarrow-\infty($ case in which $\phi=1)$ to $(\operatorname{Tr}(J), \operatorname{Det}(J))=$ $(1-2 \bar{\sigma} ;-\bar{\sigma})$, for $\phi=0$. This segment of line passes in the bifurcation point $(\operatorname{Tr}(J), \operatorname{Det}(J))=(-\bar{\sigma} / 2 ;-1+\bar{\sigma} / 2)$ as long as $\bar{\sigma}<2 / 3$.

The main difference relatively to the case of propositions 2 and 3 is that we do not encounter, in the present case, cycles with periodicity larger than 2. The region locally identified as a region of saddle-path stability is a region of period 2 cycles and, eventually, instability. An illustration follows with the bifurcation diagram in figure 10 . We let $\phi=0.25$ and present the corresponding relation between $\bar{\sigma}$ and the long run value of $x_{t}$. The steady state point is achieved for $\bar{\sigma}<6 / 11$. The bifurcation gives place to a period 2 cycle (in the example, a value $a=1$ is taken, but any other value would generate this type of dynamics).

$$
\text { *** figure } 10^{* * *}
$$

\subsection{A simple two-dimensional model}

The last two systems that will be addressed take two endogenous variables instead of one. This will transform the system under learning into a four dimensional system.

Assume variables $x_{t}, y_{t} \in \mathbb{R}$. Let the expected value of $y_{t}$ to be a real constant, $a$, and the expected value of $x_{t}$ to be the value in $t$ of $y_{t}$, i.e., 
$E_{t} x_{t+1}=y_{t}$ and $E_{t} y_{t+1}=a\left(x_{0}, y_{0}\right.$ given $)$. The corresponding steady state of the system is $\bar{x}=\bar{y}=a$.

Considering the previously used adaptive learning scheme and letting both learning processes concerning each variable to be subject to the same gain sequence, the following four dimensional system is formed,

$$
\left\{\begin{aligned}
x_{t+1} & =\frac{a}{\left[\left(1-\sigma_{t+1}\right) y_{t} / x_{t}+\sigma_{t+1} x_{t} / z_{t}\right] \cdot\left[\left(1-\sigma_{t+1}\right) a / y_{t}+\sigma_{t+1} y_{t} / v_{t}\right]} \\
y_{t+1} & =\frac{a}{\left(1-\sigma_{t+1}\right) a / y_{t}+\sigma_{t+1} y_{t} / v_{t}} \\
z_{t+1} & =x_{t} \\
v_{t+1} & =y_{t}
\end{aligned}\right.
$$

Locally, the computation of eigenvalues generates the result that follows,

Proposition 5 The system of expectations under learning that gives place to the set of equations in (9) is stable for $\bar{\sigma}<2 / 3$; otherwise, it is saddle-path stable, with a two-dimensional stable space and a two-dimensional unstable space.

Proof. Knowing that $\bar{x}=\bar{y}=a$, the linearization of system (9) around the steady state yields

$$
\left[\begin{array}{c}
x_{t+1}-\bar{x} \\
y_{t+1}-\bar{y} \\
z_{t+1}-\bar{x} \\
v_{t+1}-\bar{y}
\end{array}\right]=\left[\begin{array}{cccc}
1-2 \bar{\sigma} & -\bar{\sigma} & \bar{\sigma} & \bar{\sigma} \\
0 & 1-2 \bar{\sigma} & 0 & \bar{\sigma} \\
1 & 0 & 0 & 0 \\
0 & 1 & 0 & 0
\end{array}\right] \cdot\left[\begin{array}{l}
x_{t}-\bar{x} \\
y_{t}-\bar{y} \\
z_{t}-\bar{x} \\
v_{t}-\bar{y}
\end{array}\right]
$$

To analyze stability, one can directly compute the eigenvalues of the Jacobian matrix in (10). Two pairs of identical eigenvalues are obtained: $\lambda_{1}, \lambda_{2}=\frac{1}{2}+\frac{1}{2} \sqrt{4 \bar{\sigma}^{2}+1}-\bar{\sigma}$ and $\lambda_{3}, \lambda_{4}=\frac{1}{2}-\frac{1}{2} \sqrt{4 \bar{\sigma}^{2}+1}-\bar{\sigma}$. The first pair of eigenvalues is inside the unit circle $\forall \bar{\sigma} \in(0,1)$. The second pair may be inside or outside the unit circle; the bifurcation condition $\lambda_{3}, \lambda_{4}=1$ will not occur for the admissible values of the gain variable, but it is possible that $\lambda_{3}, \lambda_{4}=-1$; this occurs for $\bar{\sigma}=2 / 3$. Condition $\lambda_{3}, \lambda_{4}>-1$, required for stability, will imply $\bar{\sigma}<2 / 3$. Thus, if this inequality holds, the four eigenvalues lie inside the unit circle and the system is stable; in the opposite case, the first two eigenvalues are inside the unit circle, while the second pair is not: the system is saddle-path stable, with two stable dimensions and two unstable dimensions

As in the lower dimensional cases, we regard that stability exists for efficient learning processes but inefficiency causes stability to be lost. The point of bifurcation, $\bar{\sigma}=2 / 3$, is exactly the same one found in the first treated example. From a global dynamics point of view, one confirms that stability exists for $\bar{\sigma}<2 / 3$ and that, once again, the steady state value is irrelevant to the qualitative nature of the dynamics. The bifurcation generates solely period 2 cycles. Figure 11 illustrates the outcome for variable $x_{t}$.

$$
\text { *** figure } 11 * * *
$$




\subsection{A two-dimensional multiplicative model}

Our last learning model takes the following form: $E_{t} x_{t+1}=a x_{t} y_{t}$ and $E_{t} y_{t+1}=b x_{t} y_{t}$, with $x_{0}, y_{0}$ given and $a$ and $b$ any positive constants. The steady state of the system is the point $(\bar{x}, \bar{y})=(1 / b, 1 / a)$. The four dimensional system that is obtained after considering a process of adaptive learning is the following:

$$
\left\{\begin{array}{l}
x_{t+1}=\left(1-\sigma_{t+1}^{y}\right) x_{t}+\sigma_{t+1}^{y} \bar{x} \cdot \frac{y_{t}}{v_{t}} \\
y_{t+1}=\left(1-\sigma_{t+1}^{x}\right) y_{t}+\sigma_{t+1}^{x} \bar{y} \frac{x_{t}}{z_{t}} \\
z_{t+1}=x_{t} \\
v_{t+1}=y_{t}
\end{array}\right.
$$

In opposition to the assumption of the previous section, we now let the learning processes to be distinct in terms of the gain sequences. We assume the possibility of different efficiency degrees in the learning process regarding the formation of expectations of variables $x_{t}$ and $y_{t}$. In the local dynamic analysis we maintain $\overline{\sigma^{x}}=\overline{\sigma^{y}}$ in order to obtain explicit results concerning the eigenvalues of the Jacobian matrix; in the global dynamics evaluation, we will assume the general case $\overline{\sigma^{x}} \neq \overline{\sigma^{y}}$ to search for the impact of different assumptions on the quality of learning over this type of formulation.

Proposition 6 In the two-dimensional multiplicative model, under the constraint $\sigma_{t}:=\sigma_{t}^{x}=\sigma_{t}^{y}$, stability is guaranteed for $\bar{\sigma}<2 / 3$; if $\bar{\sigma}>2 / 3$, the system will be locally characterized by saddle-path stability, with three stable dimensions and one unstable dimension.

Proof. As usual, we find the linearized system and corresponding Jacobian matrix,

$$
\left[\begin{array}{c}
x_{t+1}-\bar{x} \\
y_{t+1}-\bar{y} \\
z_{t+1}-\bar{x} \\
v_{t+1}-\bar{y}
\end{array}\right]=\left[\begin{array}{cccc}
1-\bar{\sigma} & \bar{\sigma} \cdot \frac{\bar{x}}{\bar{y}} & 0 & -\bar{\sigma} \cdot \frac{\bar{x}}{\bar{y}} \\
\bar{\sigma} \cdot \bar{y} & 1-\bar{\sigma} & -\bar{\sigma} \cdot \frac{\bar{y}}{\bar{x}} & 0 \\
1 & 0 & 0 & 0 \\
0 & 1 & 0 & 0
\end{array}\right] \cdot\left[\begin{array}{c}
x_{t}-\bar{x} \\
y_{t}-\bar{y} \\
z_{t}-\bar{x} \\
v_{t}-\bar{y}
\end{array}\right]
$$

The eigenvalues associated to matrix $J$ in (12) are $\lambda_{1}, \lambda_{2}=\frac{1}{2} \cdot(1 \pm \sqrt{1-4 \bar{\sigma}})$ and $\lambda_{3}, \lambda_{4}=\frac{1}{2} \cdot\left(1 \pm \sqrt{1+4 \bar{\sigma}^{2}}\right)-\bar{\sigma}$. The first two eigenvalues lie inside the unit circle, $\forall \bar{\sigma} \in(0,1)$; these are real values if $\bar{\sigma}<1 / 4$ and complex values with a real part lower than one in modulus otherwise. The eigenvalue $\lambda_{3}=\frac{1}{2} \cdot\left(1+\sqrt{1+4 \bar{\sigma}^{2}}\right)-\bar{\sigma}$ is a positive real value lower than one, independently of $\bar{\sigma} \in(0,1)$, and therefore the only eigenvalue that may escape from the unit circle is $\lambda_{4}=\frac{1}{2} \cdot\left(1-\sqrt{1+4 \bar{\sigma}^{2}}\right)-\bar{\sigma}$. If $\bar{\sigma}<2 / 3$, then $\left|\lambda_{4}\right|<1$; if $\bar{\sigma}>2 / 3$, then $\left|\lambda_{4}\right|>1$. Four stable dimensions exist under $\bar{\sigma}<2 / 3$; a bifurcation point is $\bar{\sigma}=2 / 3$ (in this point the eigenvalue assumes the value 
-1); and for $\bar{\sigma}>2 / 3$ there are three stable dimensions and one unstable dimension (the system is saddle-path stable)

Regarding global dynamics, in this case nonlinear results are not limited to cycles of periodicity 2; irregular fluctuations (although not chaos) are found in the following illustrative example. Consider $a=10, b=8, \overline{\sigma^{x}}=$ 0.78 and let $\overline{\sigma^{y}}$ be the bifurcation parameter. Figure 12 draws a bifurcation diagram for variable $x_{t}$. One might observe that an increasing value of the steady state gain variable $\overline{\sigma^{y}}$ leads to a bifurcation that, on a first phase produces period 2 cycles but that degenerates into a region of a-periodic fluctuations. We consider $\overline{\sigma^{y}}=0.9$ to present the long run time series of $x_{t}$. The proposed values allow to evidence a-periodic motion, according to figure 13.

$$
\text { *** figures 12,13 here *** }
$$

\section{Economic Models}

In this section, we develop two applications of the adaptive learning setup that was discussed under five different prototype models. The first example refers to expectations concerning inflation; two economies are taken into account and the expectations about next period inflation in one of the economies will be dependent on the inflation level of the other economy. The second example refers to the Ramsey growth model, i.e., to the paradigm of intertemporal maximization of consumption utility subject to a resource constraint; the differences relatively to the benchmark model are two: the shadow-price of capital in the next period is not known with certainty but it is learned through time (using the notion of adaptive learning taken throughout the paper) and capital is an argument of the utility function. One of the goals of the analysis, in this second case, is to understand how one may convincingly consider that the representative agent can take utility or disutility from the existence of a given amount of capital, when the process of learning is considered.

\subsection{A two-country inflation model}

Consider two economies. The first, economy $A$, has an inflation target of $\left(\pi^{A}\right)^{*}$ which is set by the central bank. The private agents in this economy believe that, independently of the current value of inflation, in the next period the monetary authority is capable of putting inflation on the referred target, and therefore $E_{t} \pi_{t+1}^{A}=\left(\pi^{A}\right)^{*}, \pi_{0}^{A}$ given. The second economy, economy $B$, has also a defined inflation target, $\left(\pi^{B}\right)^{*}$, but the way in which expectations about future inflation are formed is not so simple as in economy $A$. On one hand, inflation expectations are dependent on the contemporaneous level of inflation in the country; on the other hand, there 
is also a dependence on the other country's inflation rate. The specification is, in concrete,

$$
E_{t} \pi_{t+1}^{B}=a_{B} \pi_{t}^{B}+a_{A}\left(\pi_{t}^{A}-\left(\pi^{A}\right)^{*}\right)+\left(1-a_{B}\right)\left(\pi^{B}\right)^{*}
$$

In equation (13), parameters $a_{A}$ and $a_{B}$ are positive values (with $a_{B} \neq$ 1). Observe that the steady state level of inflation in economy $B$ corresponds to the inflation target level, as in economy $A$.

The adaptive learning setup is assumed; for both countries an estimator as the one in section 2.1 is considered, as well as the respective dynamic process. The result is the four dimensional system that follows:

$$
\left\{\begin{aligned}
\pi_{t+1}^{A} & =\frac{\left(\pi^{A}\right)^{*}}{\left(1-\sigma_{t+1}\right) \frac{\left(\pi^{A}\right)^{*}}{\pi_{t}^{A}}+\sigma_{t+1} \frac{\pi_{t}^{A}}{z_{t}^{A}}} \\
\pi_{t+1}^{B} & =\frac{a_{A}\left(\pi_{t+1}^{A}-\left(\pi^{A}\right)^{*}\right)+\left(1-a_{B}\right)\left(\pi^{B}\right)^{*}}{\left(1-\sigma_{t+1}\right) \frac{a_{A}\left(\pi_{t}^{A}-\left(\pi^{A}\right)^{*}\right)+\left(1-a_{B}\right)\left(\pi^{B}\right)^{*}}{\pi_{t}^{B}}+\sigma_{t+1}\left(\frac{\pi_{t}^{B}}{z_{t}^{B}}-a_{B}\right)} \\
z_{t+1}^{A} & =\pi_{t}^{A} \\
z_{t+1}^{B} & =\pi_{t}^{B}
\end{aligned}\right.
$$

The question to ask in this case is whether the inflation rate in country $B$ converges to the target level or not. Under perfect foresight, the condition for stability is $a_{B} \in(-1,1)$, regardless of the value of the second parameter, $a_{A}$. Under learning the result is not that simple, as we discuss below.

Proposition 7 In the two-country inflation model, the parameters that define stability are $a_{B}$ and $\bar{\sigma}$, and, thus, the dimensionality of the stable path depends on their values. One dimension is stable independently of the values of $a_{B}$ and $\bar{\sigma}$. For $a_{B}<1$, there is another stable dimension that is independent of parameter values, while the remaining two are stable if $\bar{\sigma}<2 / 3$ and $\bar{\sigma}<2\left(1-a_{B}\right) /\left(3-a_{B}\right)$. For $a_{B}>1$, stability is guaranteed if $\bar{\sigma}<2 / 3$ and $\bar{\sigma}<a_{B}-1$.

Proof. As in the general settings, proving local stability is achievable through the computation of a linearized version of the dynamic system in consideration, in the vicinity of the steady state. In the present case, we have

$$
\left[\begin{array}{c}
\pi_{t+1}^{A}-\left(\pi^{A}\right)^{*} \\
\pi_{t+1}^{B}-\left(\pi^{B}\right)^{*} \\
z_{t+1}^{A}-\left(\pi^{A}\right)^{*} \\
z_{t+1}^{B}-\left(\pi^{B}\right)^{*}
\end{array}\right]=\left[\begin{array}{cccc}
1-2 \bar{\sigma} & 0 & \bar{\sigma} & 0 \\
-\frac{a_{A}}{1-a_{B}} \bar{\sigma} & 1-\bar{\sigma}-\frac{\bar{\sigma}}{1-a_{B}} & \frac{a_{A}}{1-a_{B}} \bar{\sigma} & \frac{\bar{\sigma}}{1-a_{B}} \\
1 & 0 & 0 & 0 \\
0 & 1 & 0 & 0
\end{array}\right] \cdot\left[\begin{array}{c}
\pi_{t}^{A}-\left(\pi^{A}\right)^{*} \\
\pi_{t}^{B}-\left(\pi^{B}\right)^{*} \\
z_{t}^{A}-\left(\pi^{A}\right)^{*} \\
z_{t}^{B}-\left(\pi^{B}\right)^{*}
\end{array}\right]
$$

The eigenvalues of the Jacobian matrix are:

$$
\lambda_{1}=\frac{1}{2\left(1-a_{B}\right)}\left[1-2 \bar{\sigma}-a_{B}(1-\bar{\sigma})-\sqrt{\left[1-2 \bar{\sigma}-a_{B}(1-\bar{\sigma})\right]^{2}+4 \bar{\sigma}\left(1-a_{B}\right)}\right]
$$




$$
\begin{aligned}
& \lambda_{2}=\frac{1}{2\left(1-a_{B}\right)}\left[1-2 \bar{\sigma}-a_{B}(1-\bar{\sigma})+\sqrt{\left[1-2 \bar{\sigma}-a_{B}(1-\bar{\sigma})\right]^{2}+4 \bar{\sigma}\left(1-a_{B}\right)}\right] \\
& \lambda_{3}=\frac{1}{2}-\bar{\sigma}-\frac{1}{2} \sqrt{4 \bar{\sigma}^{2}+1} \\
& \lambda_{4}=\frac{1}{2}-\bar{\sigma}+\frac{1}{2} \sqrt{4 \bar{\sigma}^{2}+1}
\end{aligned}
$$

Eigenvalue $\lambda_{4}$ is inside the unit circle $\forall \bar{\sigma} \in(0,1)$. Relatively to $\lambda_{3}$, this corresponds to the existence of a stable dimension if $\bar{\sigma}<2 / 3$. For the first two eigenvalues, we have to separate between cases $a_{B}<1$ and $a_{B}>1$. In case $a_{B}<1, \lambda_{2}$ is inside the unit circle independently of parameter values; $\lambda_{1}$ is inside the unit circle if $\bar{\sigma}<2\left(1-a_{B}\right) /\left(3-a_{B}\right)$. In case $a_{B}>1$, eigenvalues $\lambda_{1}, \lambda_{2}$ are a pair of eigenvalues inside the unit circle if $\bar{\sigma}<a_{B}-1$ and outside the unit circle otherwise

Stability results are, thus,

(i) Four stable dimensions: $\bar{\sigma}<2 / 3 ; \bar{\sigma}<2\left(1-a_{B}\right) /\left(3-a_{B}\right)\left[a_{B}<1\right]$ or $\bar{\sigma}<a_{B}-1\left[a_{B}>1\right]$.

(ii) Three stable dimensions: $\bar{\sigma}>2 / 3 ; \bar{\sigma}<2\left(1-a_{B}\right) /\left(3-a_{B}\right)\left[a_{B}<1\right]$ or $\bar{\sigma}<a_{B}-1\left[a_{B}>1\right]$.

(iii) Three stable dimensions: $\bar{\sigma}<2 / 3 ; \bar{\sigma}>2\left(1-a_{B}\right) /\left(3-a_{B}\right)\left[a_{B}<1\right]$.

(iv) Two stable dimensions: $\bar{\sigma}<2 / 3 ; \bar{\sigma}>a_{B}-1\left[a_{B}>1\right]$.

$(v)$ Two stable dimensions: $\bar{\sigma}>2 / 3 ; \bar{\sigma}>2\left(1-a_{B}\right) /\left(3-a_{B}\right)\left[a_{B}<1\right]$.

(vi) One stable dimension: $\bar{\sigma}>2 / 3 ; \bar{\sigma}>a_{B}-1\left[a_{B}>1\right]$.

The global dynamics analysis confirms the previous outcomes, with an additional important result. Although parameter $a_{A}$ is not relevant to determine stability, the kind of fluctuations one encounters will be dependent on the value of this parameter. Thus, the long run inflation rate in country $B$ can be influenced by how the other economy behaves regarding the path of the inflation rate. Four bifurcation diagrams are presented for the relation between $\bar{\sigma}$ and the long run value of $\pi_{t}^{B}$. The first two take $a_{B}=0.75$; the other two, $a_{B}=1.25$. The first and the third assume $a_{A}=0.1$ (a low influence of the other country's inflation on country $B$ inflation expectations); the second and the fourth take $a_{A}=0.5$. One also assumes $\left(\pi^{A}\right)^{*}=\left(\pi^{B}\right)^{*}=0.02$, although these target values are irrelevant to the qualitative dynamics.

$$
\text { *** figures } 14,15,16,17 * * *
$$

In figure 14 , the parametrization $a_{B}=0.75$ and $a_{A}=0.1$ implies that stability exists for low values of $\bar{\sigma}$ (lower than $\left.2\left(1-a_{B}\right) /\left(3-a_{B}\right)=0.222\right)$. In this specific case, the local non stability result corresponds first to a region of cycles of periodicity 2 , and higher periodicity cycles emerge for $\bar{\sigma}$ around 0.7 to 0.8 . When changing the value of $a_{A}$ to 0.5 , we get again low periodicity cycles (in this case, only period 2 cycles), but located in different positions then before (figure 15). Considering $a_{B}=1.25$, several regions of cycles of high periodicity and even chaos are depicted, both for $a_{A}=0.1$ and $a_{A}=0.5$. The areas of prevalence of chaotic motion are not, however, 
the same in the two figures (figure 16 and figure 17). In these two last cases, the point of bifurcation is $\bar{\sigma}=a_{B}-1=0.25$.

Therefore, the main result is that economy $B$ inflation is only dependent on the economy's own expectations in what regards stability properties, but it is also dependent on expectations concerning economy $A$ inflation when measuring the kind of cycles that may arise when stability does not hold.

In economic terms one verifies the complexity learning may introduce in a system otherwise simple to analyze. Cyclical motion arises, meaning that inflation does not converge necessarily to a fixed point stable outcome, as it could happen under perfect foresight; instead, the inflation rate can fluctuate around a constant value, i.e. the defined target, namely in circumstances in which the quality of learning is poor.

\subsection{The Ramsey model with capital in the utility function}

In this second economic application, we consider an optimal control growth model. Utility is maximized under a resource constraint. The constraint is the usual capital accumulation equation,

$$
k_{t+1}=f\left(k_{t}\right)-c_{t}+(1-\delta) k_{t}, k_{0} \text { given }
$$

In equation (16), $k_{t} \geq 0$ represents the amount of capital (per unit of labor) available at moment $t, c_{t} \geq 0$ is the level of consumption (also per unit of labor) in moment $t$, and $0<\delta<1$ is the capital depreciation rate. The production function is of the neoclassical type, i.e., it exhibits constant returns to scale and decreasing marginal returns, and the Inada conditions hold. Note that we present the production function in intensive form; nevertheless, we also assume that population does not grow, and therefore normalizing the labor amount to 1 , it is indifferent to refer to the variables in levels or in quantities per unit of labor. We take a Cobb-Douglas production function of the type $f\left(k_{t}\right)=A k_{t}^{\alpha}$, with $A$ a positive level of technology (or measure of the total factor productivity) and $\alpha \in(0,1)$ the output-capital elasticity.

In what concerns the utility maximization problem, we introduce an innovation relatively to the conventional specification. As usual, it is assumed that a representative agent maximizes utility intertemporally, given an infinite horizon and a discount factor $\beta \in(0,1)$, but the utility function has, as arguments, not only the consumption level but also the level of the state variable. Several interpretations can be given for this presentation: among others, one may consider that capital is mainly human capital, and that the agent withdraws utility directly from a larger amount of knowledge; differently, one can interpret the presence of capital in the utility function as something that is not advantageous to the representative agent, for instance, if more capital implies a depletion of resources, and therefore additional physical capital emerges in the utility function as a measure of 
environmental degradation. This second interpretation is the one we will see as appealing from a dynamic analysis point of view (introducing learning, nonlinear dynamics emerge in the case in which the derivative of the utility function in order to capital is a negative value).

The objective function is

$$
U_{0}=\sum_{t=0}^{+\infty} \beta^{t} U\left(c_{t}, k_{t}\right)
$$

The utility function is assumed as an additively separable function with decreasing marginal utility regarding each of the arguments; the following functional form is adopted: $U\left(c_{t}, k_{t}\right)=\ln c_{t}+m \ln k_{t}$. Parameter $m$ translates the weight of capital in terms of utility; as stated, nonlinear dynamics are obtained only for a negative impact of capital over utility, and therefore we will explore cases in which $m<0$.

The problem at hand is simply the maximization of (17) subject to (16). The difference relatively to the conventional Ramsey growth model is that, instead of perfect foresight, we assume that the representative agent learns the shadow price of capital for the time period that follows. The learning rule is the same adaptive learning procedure one has used throughout the paper.

To solve the model, one builds the Hamiltonian function, assuming $p_{t}$ as the shadow-price of capital,

$$
H\left(k_{t}, p_{t}, c_{t}\right)=\ln c_{t}+m \ln k_{t}+\beta E_{t} p_{t+1} \cdot\left[A k_{t}^{\alpha}-c_{t}-\delta k_{t}\right]
$$

The first-order optimality conditions are straightforward to obtain,

$$
\begin{gathered}
H_{c}=0 \Rightarrow 1 / c_{t}=\beta E_{t} p_{t+1} \\
E_{t} p_{t+1}-p_{t}=-H_{k} \Rightarrow\left[1-\delta+\alpha A k_{t}^{-(1-\alpha)}\right] \cdot \beta E_{t} p_{t+1}=p_{t}-m / k_{t} \\
\lim _{t \rightarrow+\infty} k_{t} \beta^{t} p_{t}=0 \text { (transversality condition) }
\end{gathered}
$$

From conditions (19) and (20), one may express the level of consumption as a function of the stock of capital and the contemporaneous price level:

$$
c_{t}=\frac{1-\delta+\alpha A k_{t}^{-(1-\alpha)}}{p_{t}-m / k_{t}}
$$

Expectations concerning the shadow-price of capital are assumed to be formed through learning. The learning estimator $b_{t}$ evolves according to the adaptive rule $b_{t}=b_{t-1}+\sigma_{t} \cdot\left(\frac{p_{t-1}}{p_{t-2}}-b_{t-1}\right), b_{0}$ given, such that $E_{t} p_{t+1}=$ 
$b_{t} p_{t}$. Thus, we can write $b_{t}=\frac{1-m /\left(p_{t} k_{t}\right)}{\beta \cdot\left[1-\delta+\alpha A k_{t}^{-(1-\alpha)}\right]}$. Replacing the estimator expression into the rule and taking in consideration the capital constraint, one arrives to the following three-dimensional system:

$$
\left\{\begin{array}{l}
k_{t+1}=A k_{t}^{\alpha}-\frac{1-\delta+\alpha A k_{t}^{-(1-\alpha)}}{p_{t}-m / k_{t}}+(1-\delta) k_{t} \\
p_{t+1}=\frac{m}{k_{t+1} \cdot\left\{1-\beta \cdot\left[1-\delta+\alpha A k_{t+1}^{-(1-\alpha)}\right] \cdot x\left(k_{t}, p_{t}, z_{t}\right)\right\}} \\
z_{t+1}=p_{t}
\end{array}\right.
$$

In system $(23)$, we define $z_{t} \equiv p_{t}$ and $x\left(k_{t}, p_{t}, z_{t}\right)=\left(1-\sigma_{t+1}\right) \cdot \frac{1-m /\left(p_{t} k_{t}\right)}{\beta \cdot\left[1-\delta+\alpha A k_{t}^{-(1-\alpha)}\right]}+$ $\sigma_{t+1} \cdot \frac{p_{t}}{z_{t}}$.

To illustrate the dynamics of the problem, we consider a numerical example and restrict the analysis to a global dynamics discussion. Note that the undertaken setup does not even allow to compute an explicit steady state set of values $(\bar{k}, \bar{p}, \bar{z}, \bar{c})$, and therefore no clear results can be inferred from a local analysis.

The following benchmark values for parameters are adopted : $A=0.25$, $\alpha=1 / 3, \beta=0.96$. Parameter $m$ will allow for nonlinear dynamics under asymptotic constant gain learning if $m<0$, that is, if the marginal utility of capital is negative; the larger the stock of capital, the lower will be the utility withdrawn by the representative agent. A possible explanation for this eventuality is that capital accumulation produces environmental degradation, and thus the larger the stock of capital, the lower will be the quality of the environment, what has a direct impact on utility. Interesting dynamic results are found, e.g., for $m=-1.5$.

In the proposed growth model, the gain variable does not emerge as a relevant bifurcation parameter. Nevertheless, by allowing other parameters to vary, namely the depreciation rate of capital, a period doubling route to chaos is evidenced. Thus, we let $\bar{\sigma}=0.75$ and take $\delta$ as the bifurcation parameter. We present two figures. Figure 18 is precisely the bifurcation diagram for variable $k_{t}$; low levels of the depreciation rate imply a period 2 cycle and, as we consider higher values for $\delta$, cycles of higher periodicity and totally a-periodic motion arise. Figure 19 displays an attractor that reveals the long run relation between capital and consumption, for a value of the depreciation rate for which chaos exists $(\delta=0.74)$. Figures 18 and 19 are drawn using the initial values $k_{0}=1, p_{0}=z_{0}=0.5$ and $c_{0}=0.2$.

$$
\text { *** figures } 18,19 * * *
$$

The dynamic features displayed in the presented figures are compatible with an intuitive reasoning concerning our growth framework: the long term values of capital and consumption fall and become less stable as the depreciation rate rises. A higher depreciation rate implies a need to generate 
additional physical capital, and this produces a negative effect over welfare, given that the marginal utility of capital is negative.

Most importantly, this exercise shows how learning can conduct to nonlinear dynamics in conventional intertemporal growth models.

\section{Conclusion}

We have developed a series of deterministic models where expectations concerning the next period value of some variable are formed through a learning process. Five generic models were presented, leading to some overall relevant conclusions: $(i)$ the steady state of the system under learning is identical to the perfect foresight steady state; $(i i)$ in each one of the models, learning introduces endogenous fluctuations, where otherwise we only encounter stability or instability; (iii) the found cycles are, in some simple models, only period 2 cycles, but higher order periodicity is also frequently found in various of the introduced models, chaotic motion is observed; $(i v)$ in the various problems, one verifies that the quality of learning matters; an asymptotic gain variable close to zero implies stability (at the perfect foresight steady state level), while cyclical motion is a result of a low quality learning process in which memory loss is relevant (implying perpetual learning). The main conclusion is that the learning process does not need to be completely efficient to obtain the goal of a stable result; this can be attained with an almost efficient learning mechanism.

From a policy analysis standpoint, the notion of an 'almost efficient learning mechanism' can be used to mitigate a relevant trade-off: to obtain the perfect foresight long run equilibrium it is not necessary to fully employ resources to eliminate any long term learning. The representative agent just has to be able to identify where the bifurcation point is located; placing herself immediately to the left of this point, the intended outcome is fulfilled. In other words, boundedly rational behavior may lead to a fully rational outcome, with the important advantage that resources needed to collect and treat information can be saved.

The two economic examples have shown the richness of results expectations under learning may produce. First, an inflation framework allowed to perceive that a simple stable result under perfect foresight is eventually transformed in an irregular cycles outcome under adaptive learning; this implies that if agents learn in order to form inflation expectations and the quality of learning is poor, then the inflation rate may fluctuate around the specified target instead of converging to the target (and therefore aggregate price volatility may be interpreted as a result of the boundedly rational behavior of economic agents, rather than the result of purely exogenous disturbances). The second example has evaluated the conventional Ramsey growth model, with capital as an argument of the utility function. In this 
case, the representative agent, that maximizes utility intertemporally, learns the shadow-price of capital through time. As a result, for some combination of values, the typical saddle-path stability result of this class of models gives place to a variety of possible fluctuations regarding the movement over time of the capital and consumption variables; in this way, one may simulate real life business cycles that are absent from the growth analysis under a plain perfect foresight scenario.

\section{References}

[1] Adam, K.; A. Marcet and J. P. Nicolini (2006). "Stock Market Volatility and Learning." European Central Bank working paper.

[2] Bullard, J. B. (1994). "Learning Equilibria." Journal of Economic Theory, vol. 64 , pp. 468-485.

[3] Bullard, J. and K. Mitra (2002). "Learning About Monetary Policy Rules." Journal of Monetary Economics, vol. 49, pp. 1105-1129.

[4] Cellarier, L. (2006). "Constant Gain Learning and Business Cycles." Journal of Macroeconomics, vol. 28, pp. 51-85.

[5] Evans, G. W. and S. Honkapohja (2001). Learning and Expectations in Macroeconomics. Princeton University Press: Princeton, New Jersey.

[6] Evans, G. W. and S. Honkapohja (2008). "Expectations, Learning and Monetary Policy: an Overview of Recent Research." Centre for Dynamic Macroeconomic Analysis Working Paper Series, CDMA 08/02.

[7] Gauthier, S. (2002). "Determinacy and Stability under Learning of Rational Expectations Equilibria." Journal of Economic Theory, vol. 102, pp. 354-374.

[8] Giannitsarou, C. (2003). "Heterogeneous Learning." Review of Economic Dynamics, vol. 6, pp. 885-906.

[9] Hommes, C. and G. Sorger (1998). "Consistent Expectations Equilibria." Macroeconomic Dynamics, vol. 2, pp. 287-321.

[10] Honkapohja, S. and K. Mitra (2003). "Learning with Bounded Memory in Stochastic Models." Journal of Economic Dynamics and Control, vol. 27, pp. 1437-1457.

[11] Honkapohja, S. and K. Mitra (2006). "Learning Stability in Economies with Heterogeneous Agents." Review of Economic Dynamics, vol. 9, pp. 284-309. 
[12] Lucas, R. E. (1972). "Expectations and the Neutrality of Money." Journal of Economic Theory, vol. 4, pp. 103-124.

[13] Marcet, A. and T. Sargent (1989). "Convergence of Least Squares Learning Mechanisms in Self-referential Linear Stochastic Models." Journal of Economic Theory, vol. 48, pp. 337-368.

[14] Muth, J. F. (1961). "Rational Expectations and the Theory of Price Movements." Econometrica, vol. 29, pp. 315-335.

[15] Orphanides, A. and J. C. Williams (2007). "Robust Monetary Policy with Imperfect Knowledge." Journal of Monetary Economics, vol. 54, pp. 1406-1435.

[16] Schonhofer, M. (1999). "Chaotic Learning Equilibria.", Journal of Economic Theory, vol. 89, pp. 1-20.

[17] Sobel, J. (2000). "Economists' Models of Learning." Journal of Economic Theory, vol. 94, pp. 241-261.

[18] Sorger, G. (1998). "Imperfect Foresight and Chaos: an Example of a Self-Fulfilling Mistake." Journal of Economic Behaviour and Organization, vol. 33, pp. 333-362. 


\section{Figures}

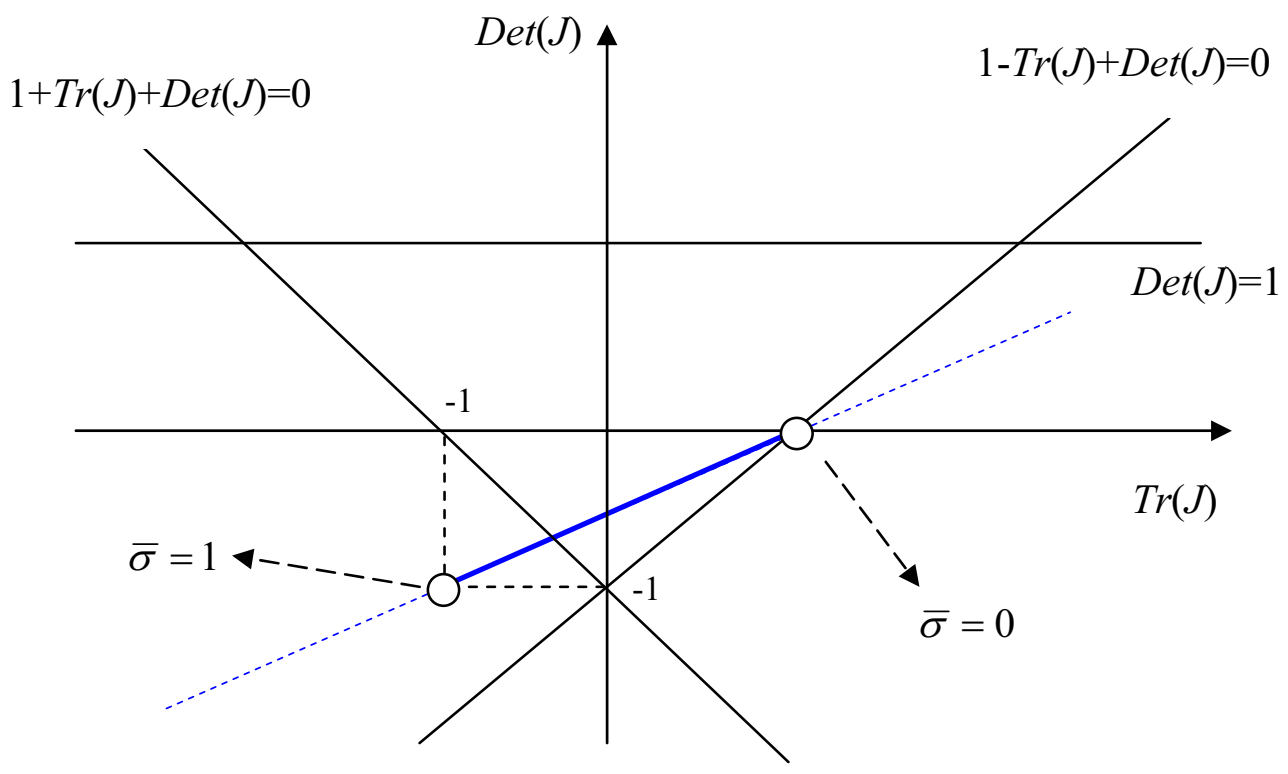

Figure 1 - Model 2.1: local dynamics.

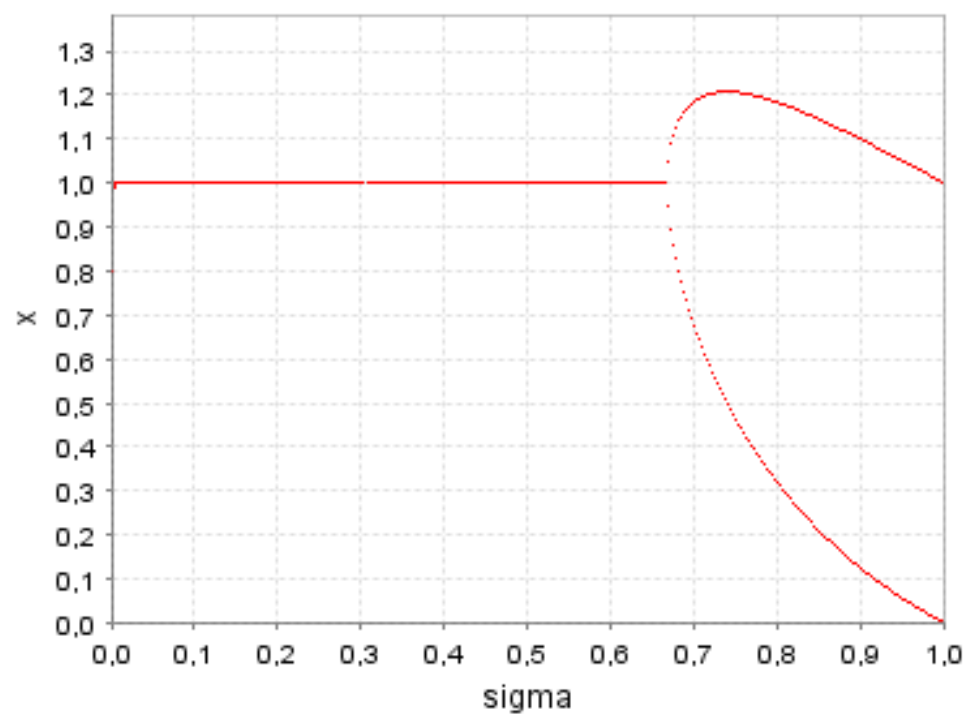

Figure 2 - Model 2.1: bifurcation diagram. 


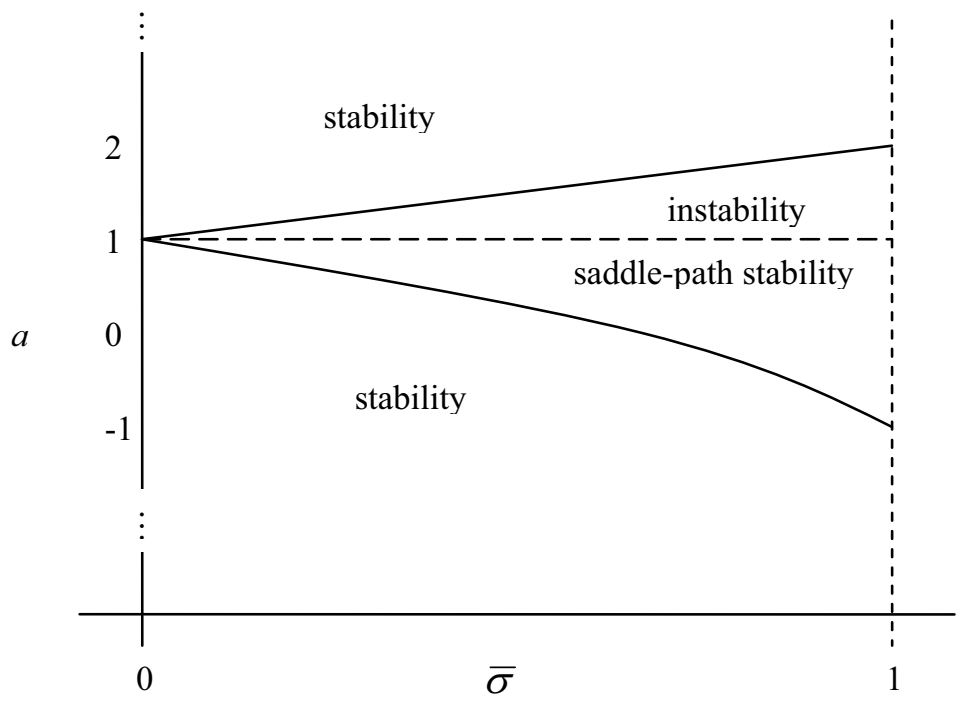

Figure 3 - Model 2.2: local dynamics; stability regions in the space of parameters.

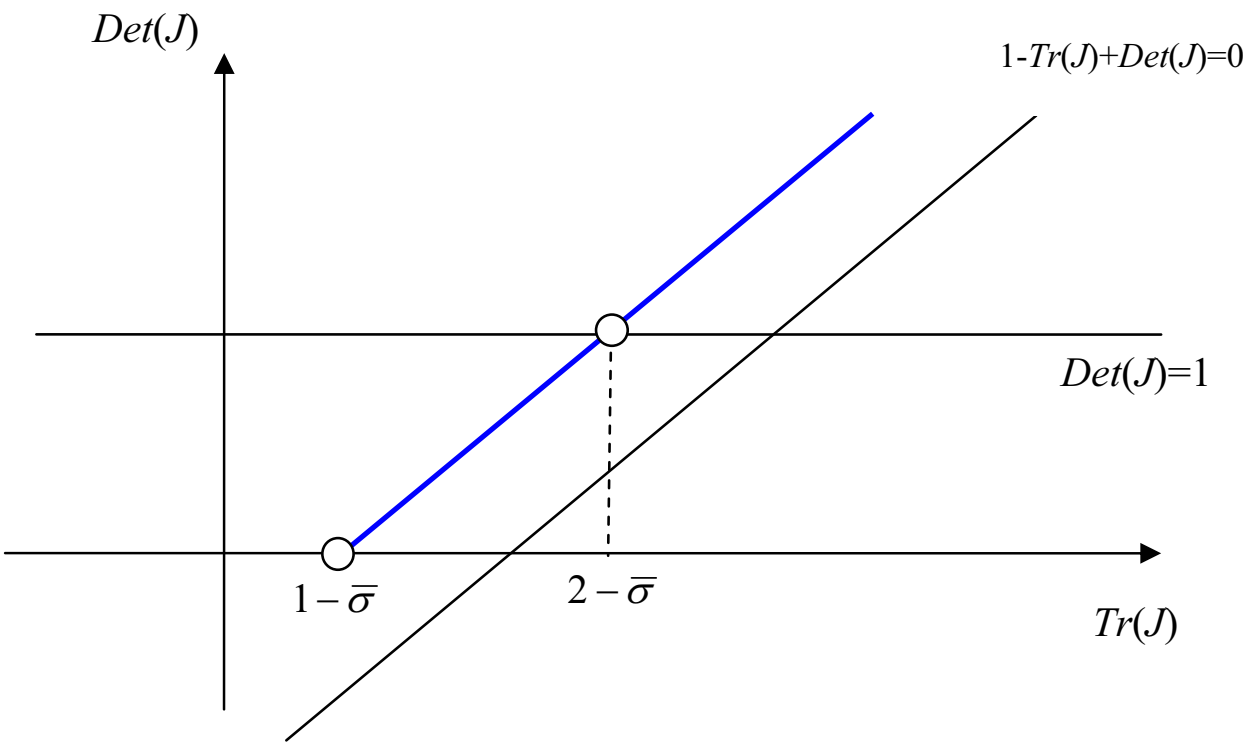

Figure 4 - Model 2.2: local dynamics; trace-determinant relation, for $a>1$. 


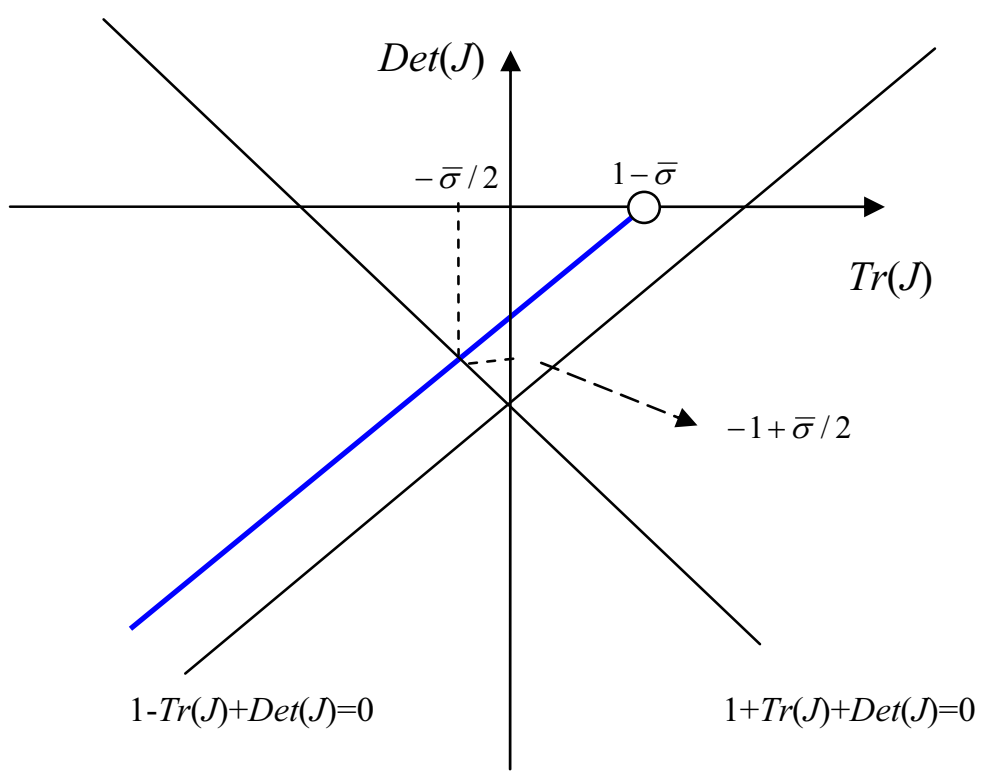

Figure 5 - Model 2.2: local dynamics; trace-determinant relation, for $a<1$.

\begin{tabular}{|c|c|}
\hline$a ; \bar{\sigma}$ & Largest LCE \\
\hline $0.85 ; 0.994$ & 0.523 \\
\hline $0.95 ; 0.765$ & 0.232 \\
\hline $1.15 ; 0.387$ & 0.496 \\
\hline $1.25 ; 0.790$ & 0.390 \\
\hline
\end{tabular}

Table 1 - Model 2.2: Largest Lyapunov characteristic exponent (examples of parameter combinations that imply chaos). 


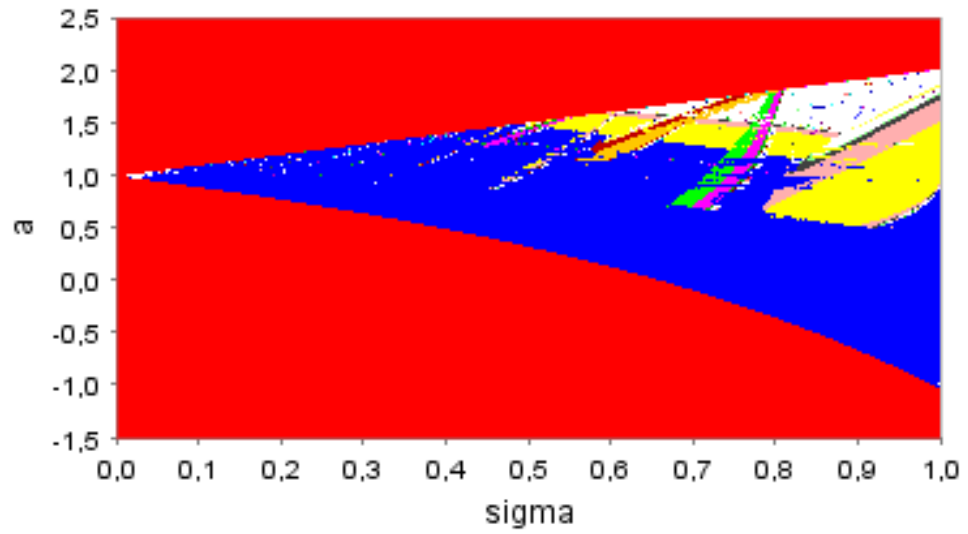

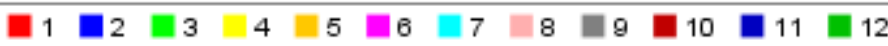

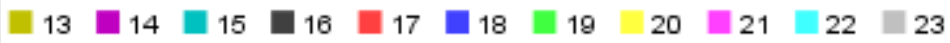

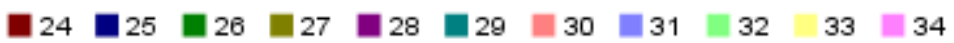

$35>35 \square$ infinity

Figure 6 - Model 2.2: global dynamics in the space of parameters.

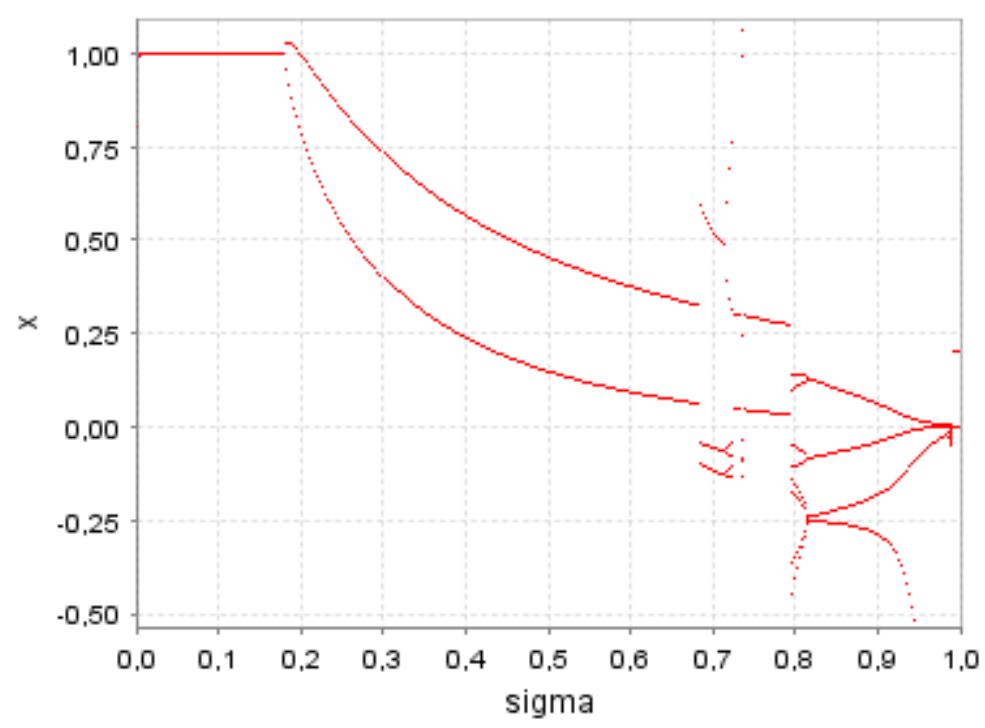

Figure 7 - Model 2.2: bifurcation diagram $(\mathrm{a}=\mathbf{0 . 8})$. 


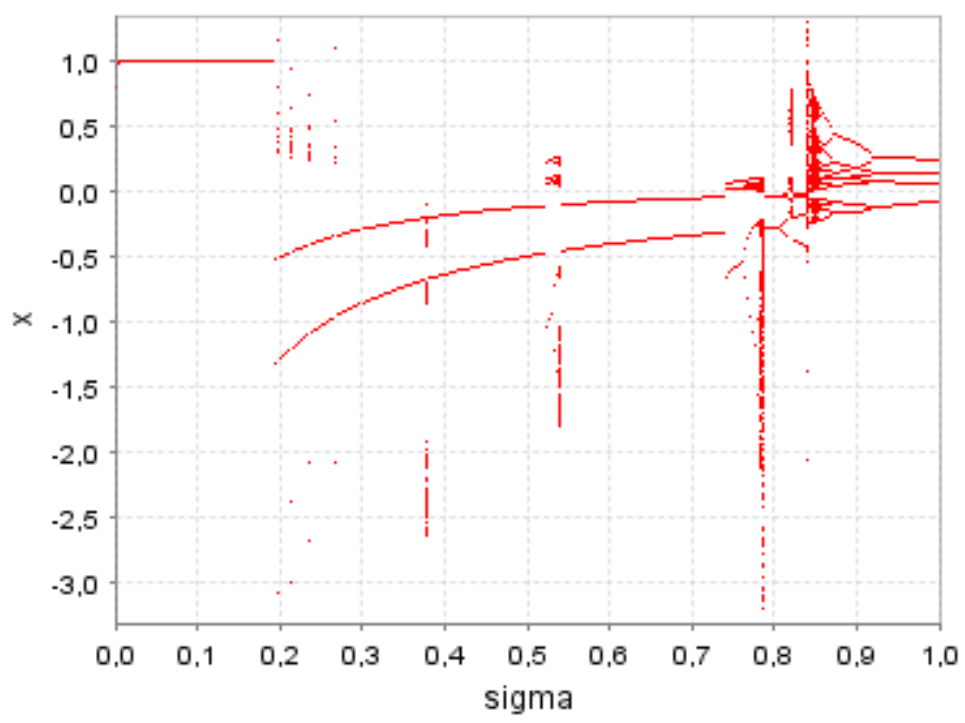

Figure 8 - Model 2.2: bifurcation diagram ( $\mathrm{a}=1.2)$.

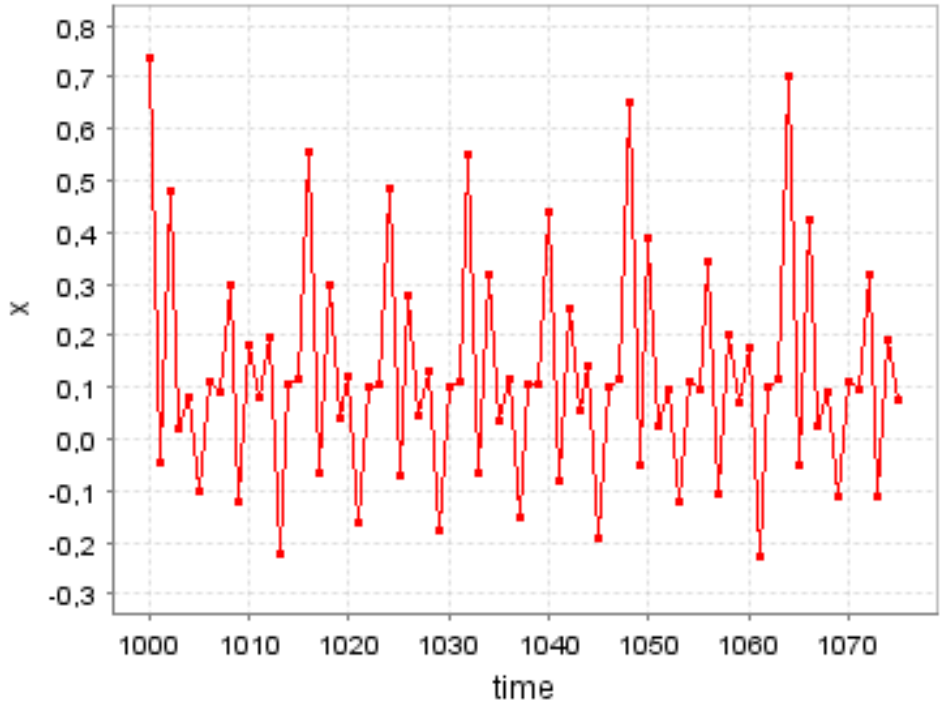

Figure 9 - Model 2.2: time series of $x_{t}(\mathrm{a}=1.2 ; \sigma=0.8475)$. 


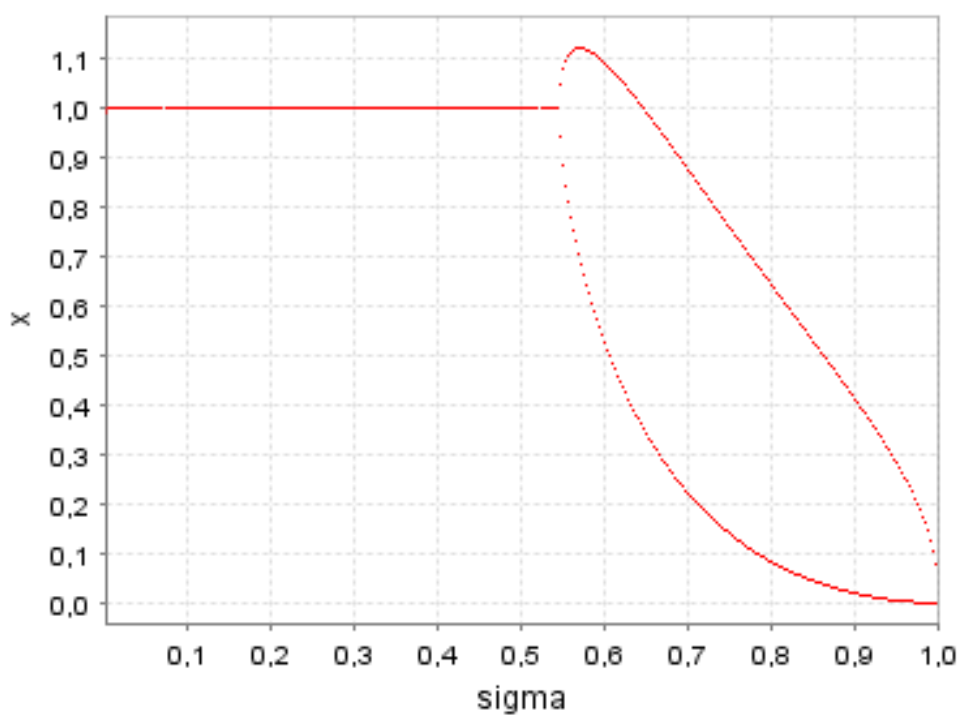

Figure 10 - Model 2.3 : bifurcation diagram.

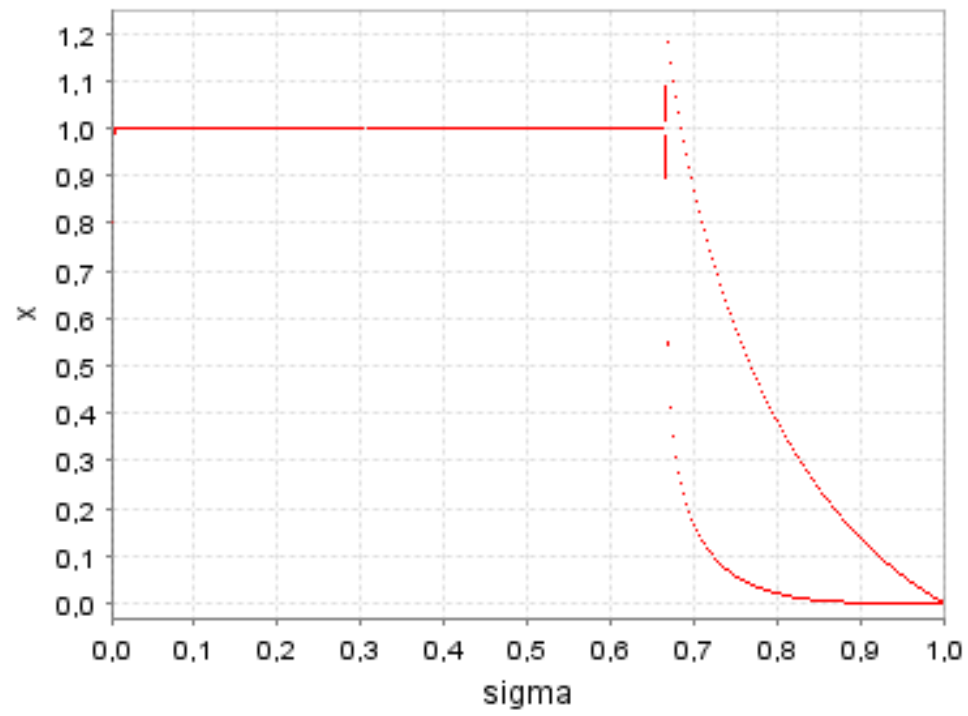

Figure 11 - Model 2.4: bifurcation diagram $\left(x_{t} ; \sigma\right)$. 


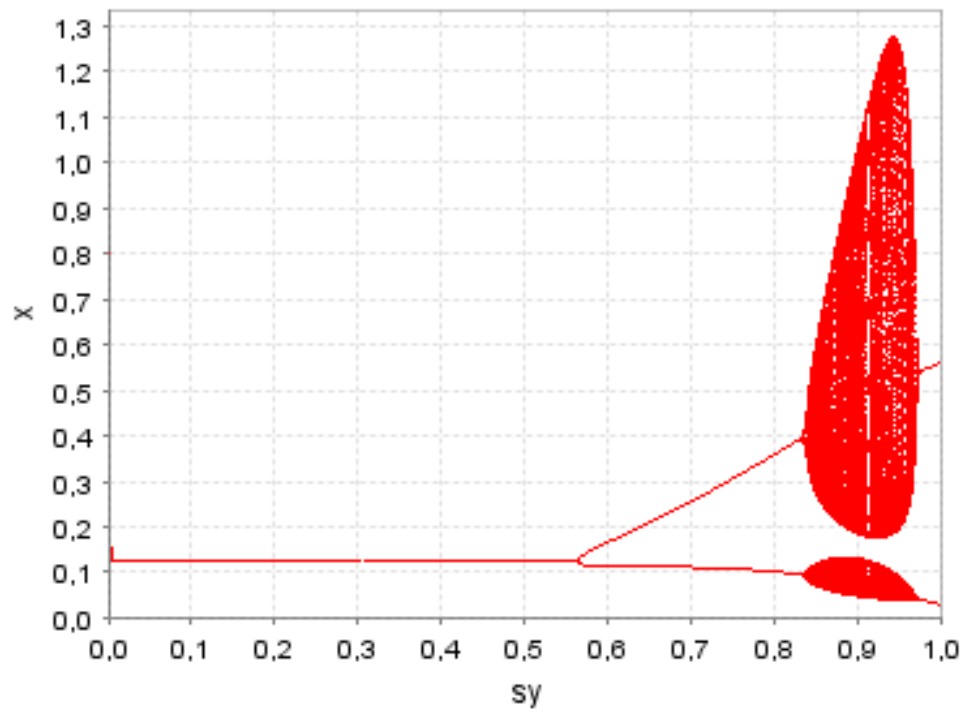

Figure 12 - Model 2.5: bifurcation diagram $\left(x_{t} ; \sigma^{\prime}\right)$.

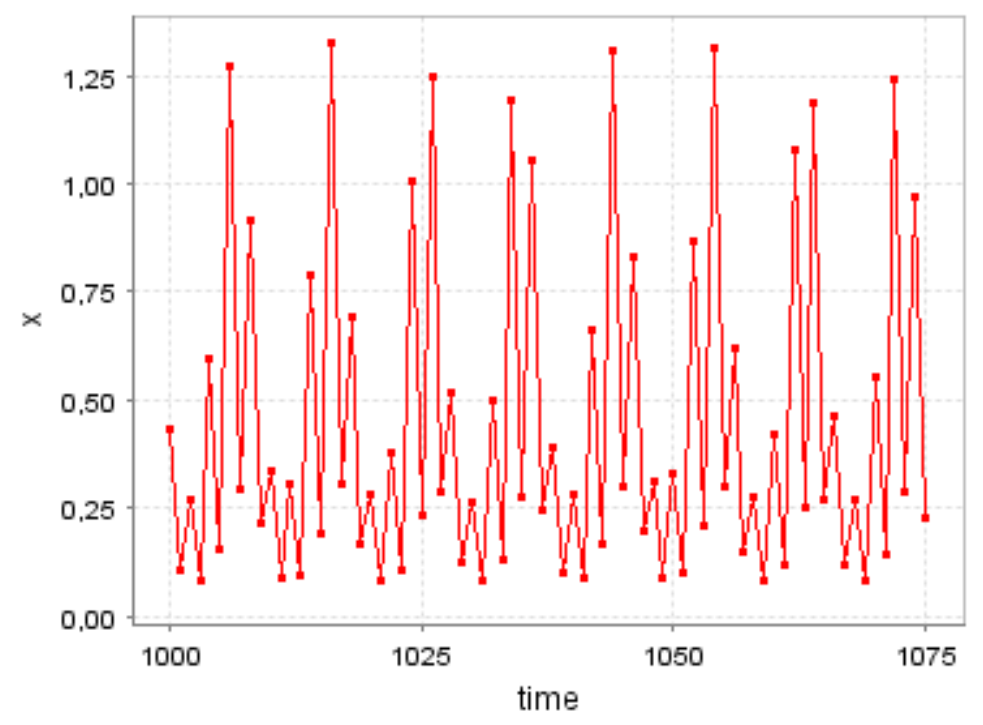

Figure 13 - Model 2.5: long term time series of $x_{t}$. 


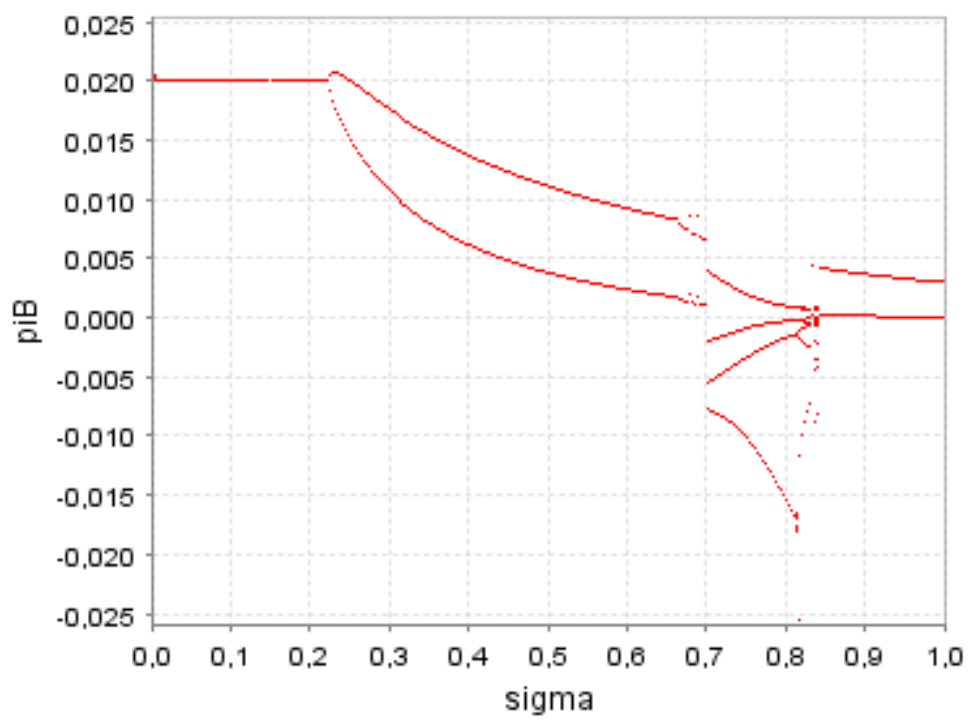

Figure 14 - Model 3.1: bifurcation diagram $\left(a_{A}=0.1 ; a_{B}=0.75\right)$.

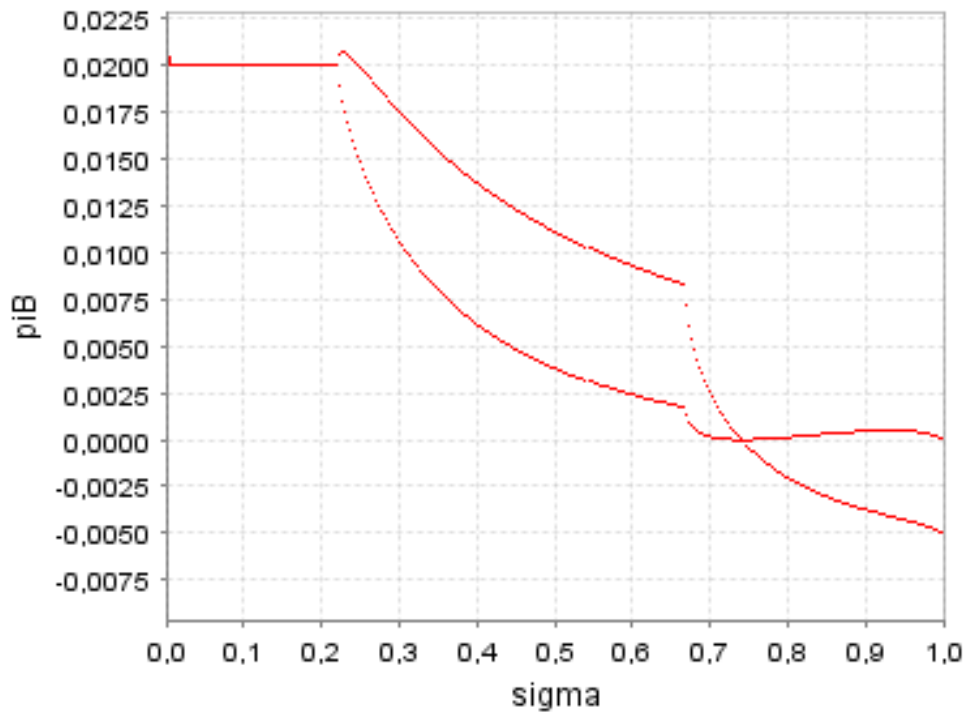

Figure 15 - Model 3.1: bifurcation diagram $\left(a_{A}=0.5 ; a_{B}=0.75\right)$. 


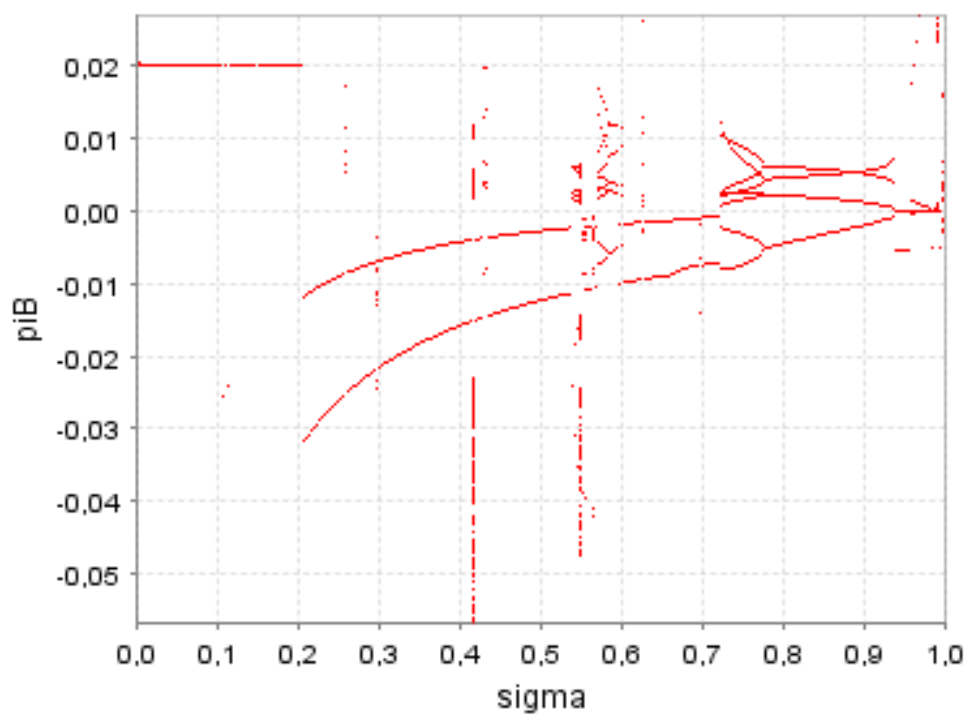

Figure 16 - Model 3.1: bifurcation diagram $\left(a_{A}=0.1 ; a_{B}=1.25\right)$.

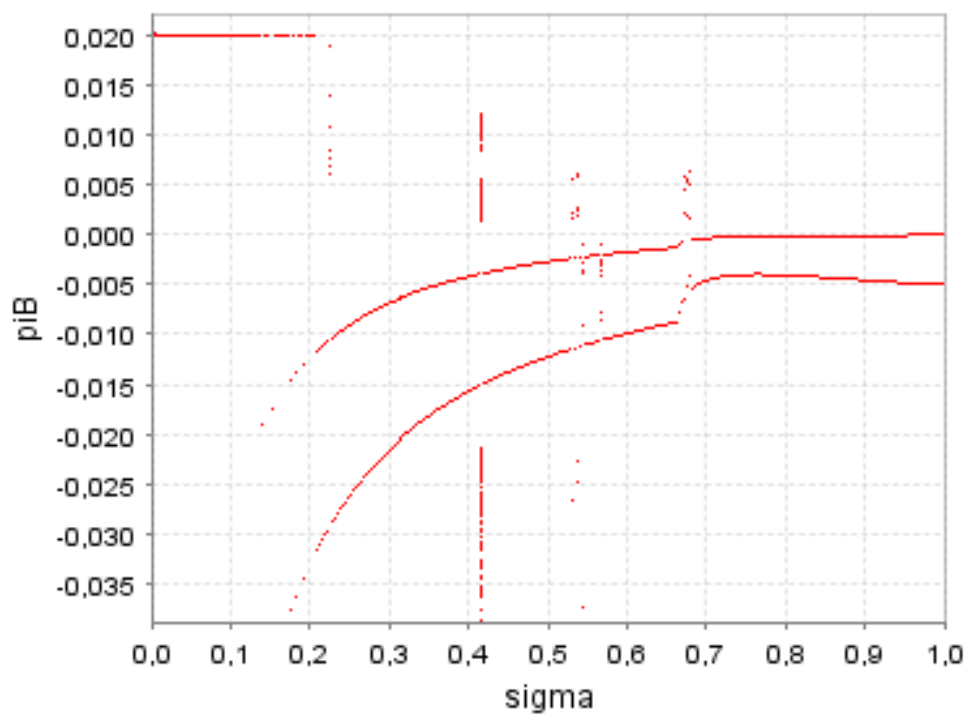

Figure 17 - Model 3.1: bifurcation diagram $\left(a_{A}=0.5 ; a_{B}=1.25\right)$. 


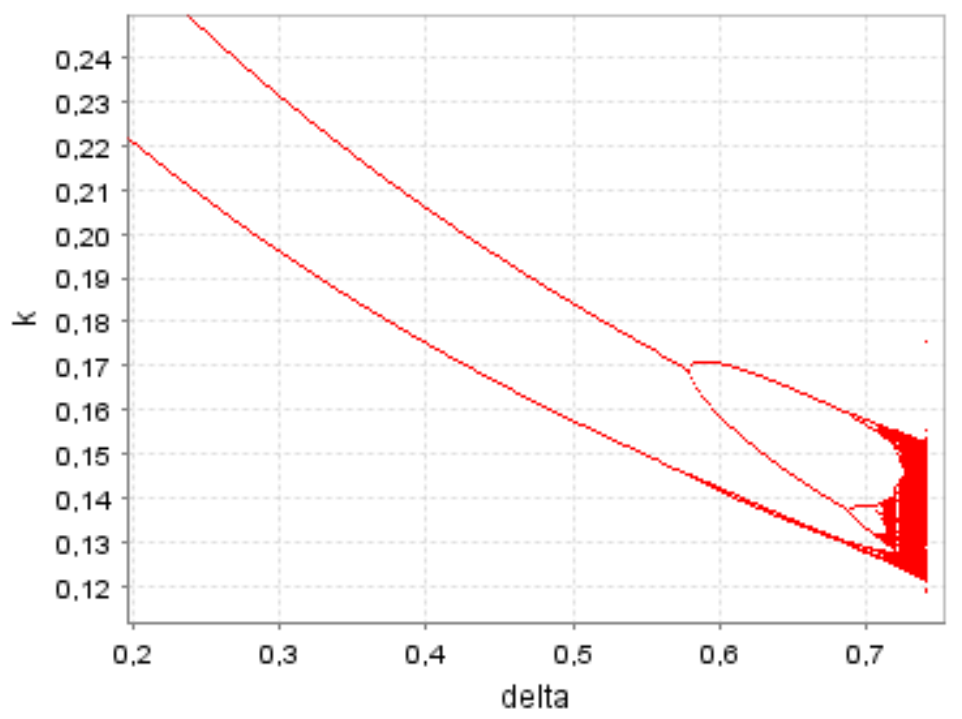

Figure 18 - Model 3.2: bifurcation diagram (bifurcation parameter: $\delta$ ).

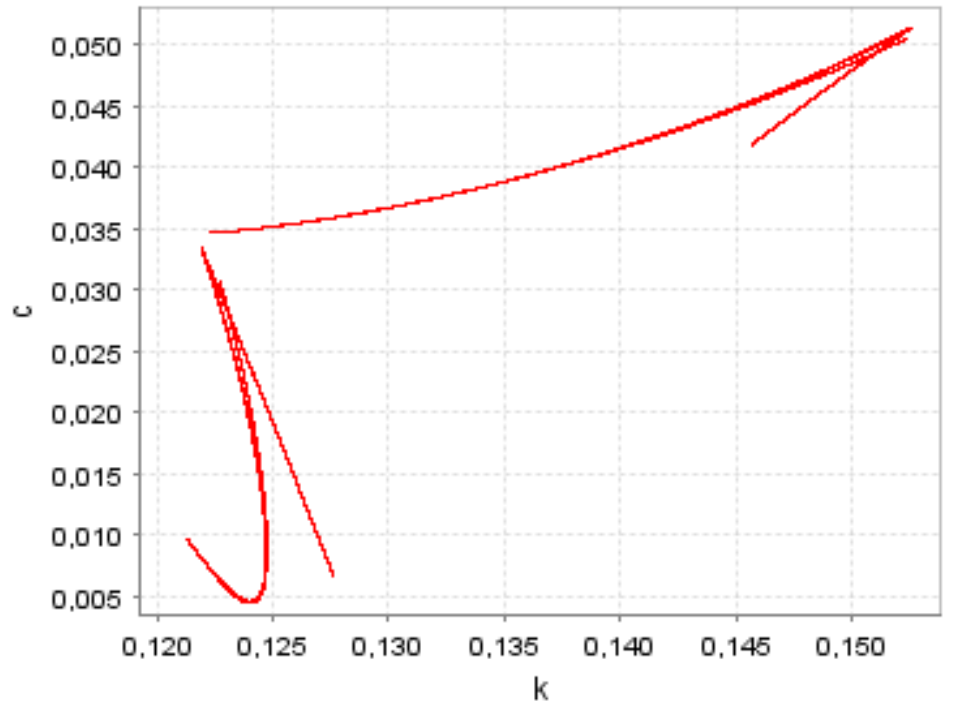

Figure 19 - Model 3.2: long term attractor $\left(k_{t} ; c_{t}\right) ; \delta=0.74$. 